\title{
Pleiades low-mass brown dwarfs: the cluster $L$ dwarf sequence
}

\author{
G. Bihain ${ }^{1,2}$, R. Rebolo ${ }^{1,2}$, V. J. S. Béjar ${ }^{1,3}$, J. A. Caballero ${ }^{1}$, C. A. L. Bailer-Jones ${ }^{4}$, \\ R. Mundt ${ }^{4}$, J. A. Acosta-Pulido ${ }^{1}$, and A. Manchado Torres ${ }^{1,2}$
}

\author{
1 Instituto de Astrofísica de Canarias, c/ Vía Láctea, s/n, 38205 La Laguna, Tenerife, Spain \\ e-mail: [gbihain;rrl;zvezda;jap;amt]@1l.iac.es;victor.bejar@gtc.iac.es \\ 2 Consejo Superior de Investigaciones Científicas, Spain \\ 3 GTC Project, Instituto de Astrofísica de Canarias \\ 4 Max-Planck-Institut für Astronomie, Königstuhl 17, 69117 Heidelberg, Germany \\ e-mail: [calj ; mundt] @mpia-hd.mpg.de
}

Received 1 March 2006 / Accepted 3 August 2006

\begin{abstract}
Aims. We present a search for low-mass brown dwarfs in the Pleiades open cluster. The identification of Pleiades members fainter and cooler than those currently known allows us to constrain evolutionary models for L dwarfs and to extend the study of the cluster mass function to lower masses.

Methods. We conducted a $1.8 \mathrm{deg}^{2}$ near-infrared $J$-band survey at the $3.5 \mathrm{~m}$ Calar Alto Telescope, with completeness $J_{\text {cpl }} \sim 19.0$. The detected sources were correlated with those of previously available optical $I$-band images $\left(I_{\mathrm{cpl}} \sim 22\right)$. Using a $J$ versus $I-J$ colour-magnitude diagram, we identified 18 faint red L-type candidates, with magnitudes $17.4<J<19.7$ and colours $I-J>3.2$. If Pleiades members, their masses would span $\sim 0.040-0.020 M_{\odot}$. We performed follow-up $H K_{\mathrm{s}}$-band imaging to further confirm their cluster membership by photometry and proper motion.

Results. Out of $11 I J$ candidates with proper motion measurements, we find six cluster members, two non-members and three whose membership is uncertain and depends on the intrinsic velocity dispersion of Pleiades brown dwarfs. This dispersion $\left(>4\right.$ mas $\left.\mathrm{yr}^{-1}\right)$ is at least four times that of cluster stars with masses $\gtrsim 1 M_{\odot}$. Five of the seven other $I J$ candidates are discarded because their $J-K_{\mathrm{s}}$ colours are bluer than those of confirmed members. Our least massive proper motion members are BRB 28 and 29 ( $\left.\sim 25 M_{\text {Jup }}\right)$. The $J$ versus $I-J$ sequence of the L-type candidates at $J>18$ is not as red as theoretical models predict; it rather follows the field L-dwarf sequence translated to the cluster distance. This sequence overlapping, also observed in the $J$ versus $J-H$ and $J-K$ diagrams, suggests that Pleiades and field L dwarfs may have similar spectral energy distributions and luminosities, and thus possibly similar radii. Also, we find $\alpha=0.5 \pm 0.2$ for a power-law approximation $\mathrm{d} N / \mathrm{d} M \propto M^{-\alpha}$ of the survey mass spectrum in the mass range $0.5-0.026 M_{\odot}$. This value is similar to that of much younger clusters, indicating no significant differential evaporation of low-mass Pleiades members relative to more massive ones.
\end{abstract}

Key words. open clusters and associations: individual: Pleiades - stars: low-mass, brown dwarfs stars: luminosity function, mass function

\section{Introduction}

As a result of progressive cooling, brown dwarfs are expected to evolve from late $\mathrm{M}$ spectral type at very early stages (age $\lesssim 10 \mathrm{Myr}$ ) to L-type ( $T_{\text {eff }}$ in the range $\sim 2400$ to $\sim 1400 \mathrm{~K}$; Dahn et al. 2002), and at sufficiently old ages (a few Gyr), to T-type and beyond ( $T_{\text {eff }} \lesssim 1400 \mathrm{~K}$ ). The mass-spectral type and mass-luminosity relationships at different ages remain to be derived from observations of cool dwarfs, which also permit the calibration of the theory of substellar evolution (e.g. Chabrier \& Baraffe 2000). In comparison to the spectra of $M$ dwarfs, $\mathrm{L}$ dwarfs are characterized in the optical by the weakening of the metal-oxide $\mathrm{TiO}$ and $\mathrm{VO}$ bands due to the condensation of the metals $\mathrm{Ti}$ and $\mathrm{V}$ in dust grains that may significantly affect the atmospheric structure and the emergent spectrum (e.g. the DUSTY models from Chabrier et al. 2000). Bands of metalhydrides $\mathrm{CrH}, \mathrm{FeH}$ and $\mathrm{CaH}$ and bands of water become more intense especially in the near-infrared (IR), whereas the neutral alkali metals $\mathrm{Na}, \mathrm{K}, \mathrm{Rb}, \mathrm{Cs}$ and $\mathrm{Li}$ become stronger in the optical (Chabrier \& Baraffe 2000; Basri 2000). The emergence in the near-IR of $\mathrm{CH}_{4}$ bands characterizes the beginning of the T dwarf sequence. Field L dwarfs have $I-J$ and $J-K_{\mathrm{s}}$ colours redder than $\sim 3.3$ and $\sim 1.3 \mathrm{mag}$, respectively, increasing from early to later spectral subclasses (Martín et al. 1999). For the L4-L8 spectral subclasses the near-IR $J-H$ and $J-K_{\mathrm{S}}$ colours appear to saturate at $\sim 1.2$ and $\sim 2.0$ mag (Kirkpatrick et al. 2000; Burgasser et al. 2002), respectively, whereas for T dwarfs these colours become bluer, with values decreasing to below zero (Knapp et al. 2004). From L0 to T8 spectral types the $I-J$ colour is increasing to $~ 5.8 \mathrm{mag}$ (Dahn et al. 2002). Associated with the other colours it can give a rough indication of the spectral type.

The Pleiades open cluster $(\sim 120 \mathrm{Myr}, \sim 130 \mathrm{pc})$ served for decades as a reference stellar laboratory where models were contrasted with observations. Since the discovery of Pleiades brown dwarfs (Rebolo et al. 1995, 1996), subsequent studies have provided numerous fainter substellar candidates with spectral types down to late M (Zapatero Osorio et al. 1997a,b; Bouvier et al. 1998, hereafter B98; Festin 1998a; Zapatero Osorio et al. 1999), from which a fraction is confirmed by lithium detection (Stauffer et al. 1998b; Martín et al. 2000) or by proper motion (Moraux et al. 2001, hereafter M 01). Very large area proper motion surveys (Hambly et al. 1999; Deacon \& Hambly 2004) have obtained on the other hand a significant 
census of the stellar-substellar boundary population of the cluster. The coolest object for which a spectral type has been obtained is the L0 brown dwarf candidate Roque 25 (Martín et al. $1998 \mathrm{~b}$ ), with an estimated theoretical mass of $\sim 0.035 M_{\odot}$. A dozen fainter objects than Roque 25 have been identified in deep surveys (Festin 1998a; Béjar 2000; Dobbie et al. 2002; Nagashima et al. 2003; Moraux et al. 2003, hereafter M03; Schwartz \& Becklin 2005), and some were confirmed by proper motion (Bouy et al. 2006).

The mass spectrum $\mathrm{d} N / \mathrm{d} M=f(M)$ of the Pleiades cluster, where $\mathrm{d} N$ stands for the number of objects in the mass range $\mathrm{d} M$, is best fitted by a lognormal function (M03; Deacon \& Hambly 2004), and its approximation by a power law $M^{-\alpha}$ in the low mass range $0.6-0.03 M_{\odot}$ provides a spectral index $\alpha \sim 0.6-0.8$ (Dobbie et al. 2002; M03). Similar values are found in other much younger open clusters, as for example $\sigma$ Orionis $(\sim 2-4 \mathrm{Myr})$, where $\alpha=0.8 \pm 0.4$ for $0.2<M\left(M_{\odot}\right)<0.013$ (Béjar et al. 2001), and IC 348 ( 3 Myr), where $\alpha=0.7 \pm 0.2$ for $0.5<M\left(M_{\odot}\right)<0.035$ (Tej et al. 2002). It is important to determine the behaviour of the $\alpha$ index in the lower mass domain because it can help to discriminate among brown dwarf formation mechanisms. For instance, for the formation of gravitationally unstable cores by turbulent fragmentation (Padoan \& Nordlund 2004), the mass spectrum is predicted to be log-normal, with a peak that appears at smaller masses when the sonic Mach number and the mean density of the cores are greater.

In this paper we present a $1.8 \mathrm{deg}^{2}$ deep $J$-band survey of the Pleiades open cluster aimed to find lower-mass brown dwarfs $\left(<0.035 M_{\odot}\right)$, by comparison with a new analysis of $I$-band data obtained by B98. Our goal is to identify L dwarf cluster candidates and study the location of the L dwarf sequence in the colour-magnitude diagram. Preliminary results have been reported in Bihain et al. (2005). Because the cluster has a well known age, the properties of Pleiades L-type brown dwarfs could provide useful constraints to models predicting the evolution of substellar objects. Additionally, we can estimate the cluster mass spectrum of the surveyed area at lower masses.

\section{Observations and data reduction}

\subsection{J-band survey}

We obtained $J$-band images on 1998 October 27-28 with the Omega-Prime $\left(\Omega^{\prime}\right)$ instrument at the $3.5 \mathrm{~m}$ Telescope of the Centro Astronómico Hispano Alemán (CAHA; Calar Alto, Spain). The fields were specifically chosen to cover the optical $R I$-band survey from B98 obtained with the $3.6 \mathrm{~m}$ CanadaFrance-Hawaii Telescope (CFHT)/UH8K survey. We observed the fields CFHT 2, 5 (north half), 6, 7, 8, 10, 11 and 13 (see their spatial distribution in Fig. 1). They correspond to an area of the cluster of $\sim 1.8$ square degrees. The $\Omega^{\prime}$ camera has a $1024 \times 1024$ $\mathrm{HgCdTe}$ Hawaii detector with a pixel scale of $0.40^{\prime \prime} \mathrm{pixel}^{-1}$ and a field of view of $6.8^{\prime} \times 6.8^{\prime}$. To cover each of the $29^{\prime} \times 29^{\prime} \mathrm{UH} 8 \mathrm{~K}$ fields (hereafter CFHT fields, as designed in B98) a connecting observation macro was used. The exposure times and repetitions in each pointing were $16 \mathrm{~s} \times 2$, except for CFHT7 and CFHT8 $(16 s \times 3)$, and CFHT6 $(18 s \times 2)$. Because the offset between successive macro pointings corresponded to one third of an $\Omega^{\prime}$ field of view, each area was repeated in total 6-9 times (96-144 s), depending of the CFHT field. The individual images were skysubtracted, flat-fielded using dome flats, combined and aligned in horizontal strips of $\sim 30^{\prime} \times 6.8^{\prime}$, using standard scripts and

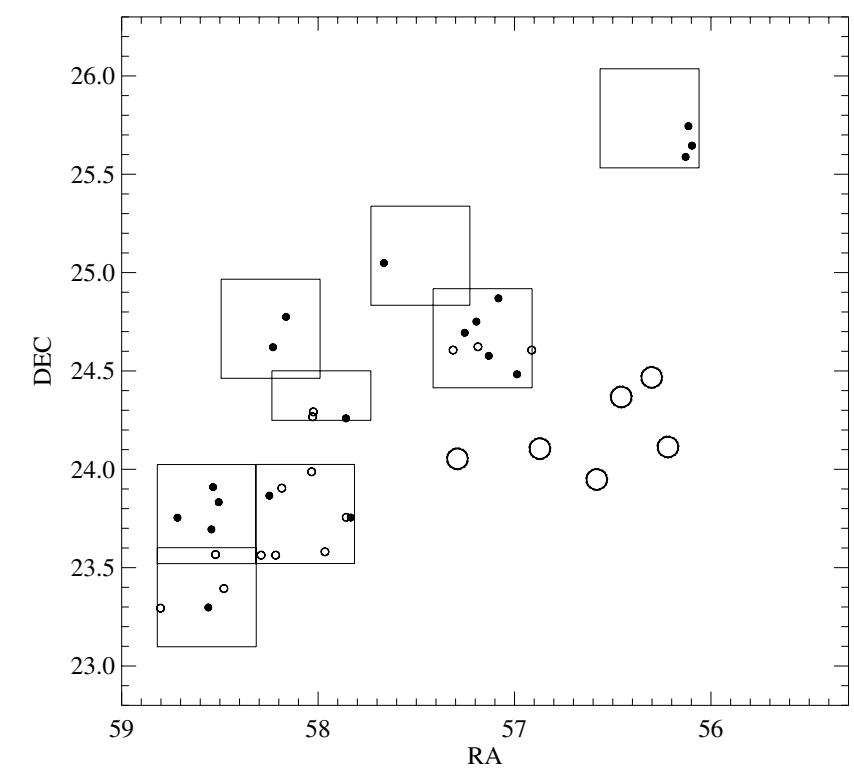

Fig. 1. Location of the surveyed Pleiades fields (squares) relative to the brightest Pleiades members with visual magnitude $V<5$ (large open circles). The small open circles represent the brown dwarf candidates from B98 with lithium or proper motion consistent with cluster membership, and the filled circles represent all the other candidates identified in this study.

routines within the $\operatorname{IRAF}^{1}$ environment. These were later analyzed for aperture and PSF photometry using DAOPHOT. The PSF photometry was calibrated with measurements from the Two Micron All Sky Survey (2MASS) Point Source Catalog (Cutri et al. 2003) and which had errors of less than 0.1 mag. On average, about five 2MASS calibrators were used per horizontal strip. The $J$-band completeness (limiting) magnitude was estimated at the maximum (fainter half maximum) of the histogram of object counts per magnitude. We found values of $\sim 19.0$ and $\sim 19.5 \mathrm{mag}$, respectively.

The optical survey in the RI bands from B98 was obtained with the UH8K instrument at the CFHT (Mauna Kea, Hawai'i) on 1996 December 9-13. The UH8K camera comprises eight Loral $2048 \times 4096$ CCD detectors with a pixel scale of $0.21^{\prime \prime}$ pixel $^{-1}$. These data were used initially for a study of brown dwarfs and low-mass stars by B98. In the present paper we use an independent data analysis by Béjar (2000). The photometric calibration of the CFHT fields was performed by comparing our photometry to the Cousins photometry of their candidates and applying an average offset; for the fields CFHT6 and CFHT8 however, different offsets were applied because they were observed through thin cirrus. The completeness and limiting magnitudes were $\sim 23.5$ and $\sim 25.0$ mag in the $R$ band, and $\sim 22.5$ and $\sim 23.5 \mathrm{mag}$ in the $I$ band, respectively.

To identify fainter and redder objects than those found by B98 and Béjar (2000), we used as reference the detected $J$-band sources and searched for their optical counterparts in the I-band images using a FORTRAN correlation program, CORREL, kindly provided by M. R. Zapatero Osorio. The correlated objects were plotted in a $J$ versus $I-J$ diagram (see Fig. 2) and contrasted with the 120 Myr DUSTY isochrone from Chabrier et al. (2000), shifted to the cluster distance of $133.8 \pm 3.0 \mathrm{pc}$

1 IRAF is distributed by the National Optical Astronomy Observatories, which are operated by the Association of Universities for Research in Astronomy, Inc., under cooperative agreement with the National Science Foundation. 


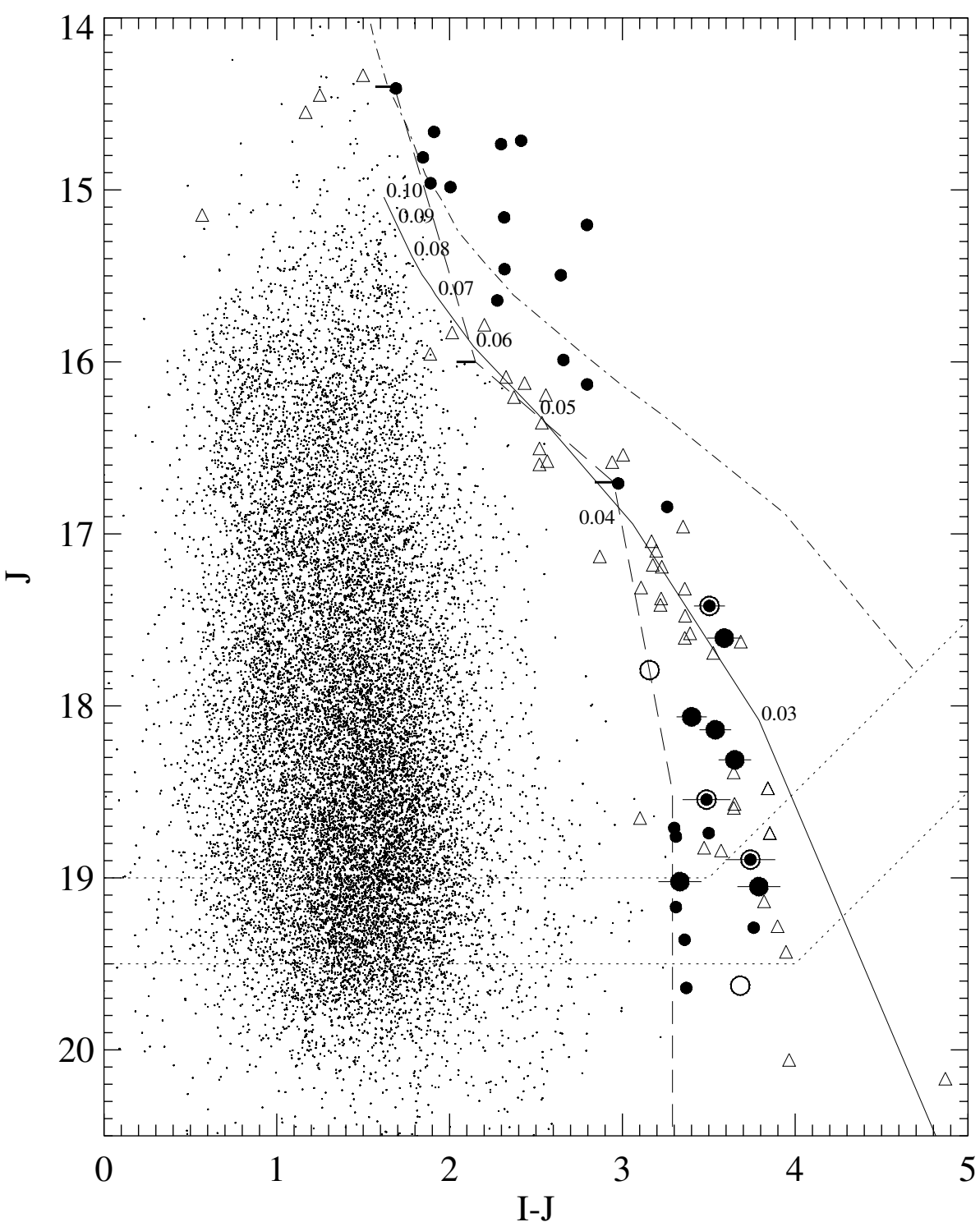

Fig. 2. $J$ versus $I-J$ colour-magnitude diagram for the correlated survey objects. The circular symbols correspond to $I J$ candidate or confirmed cluster members (Table 1), with redder $I-J$ colours than the dashed line boundary (see text for details). At $J>17.4$ we plot: proper motion members (large filled circles), possible proper motion members (circled filled circles) and non-proper motion members (large empty circles). The solid line and the dash-dotted line represent the $\sim 120 \mathrm{Myr}$ DUSTY isochrone (Chabrier et al. 2000) and the $\sim 125 \mathrm{Myr}$ NextGen isochrone (Baraffe et al. 1998), respectively, shifted to the distance of the cluster. Masses in solar units are indicated for the DUSTY isochrone. The triangles correspond to field dwarfs shifted to the distance of the cluster. Finally, the upper and lower dotted lines at the bottom of the diagram indicate the completeness and limiting magnitudes of the survey, respectively.
(Percival et al. 2005). As most of our faint objects were bluer than the theoretical isochrone in the $J$ versus $I-J$ diagram, we decided to establish our selection of candidates based on an empirical criterion. We considered a bluer envelope to cluster candidates (dashed line in Fig. 2), defined by the bluest lithium or proper motion Pleiades objects reported (Martín et al. 2000; M 01): CFHT-Pl-1, Calar 1 and CFHT-Pl-25 (horizontal thick marks in Fig. 2). The envelope was extended to faint magnitudes $J>18.5$ at a fixed colour of $I-J=3.3$, to avoid most of the M8-9 dwarf contaminants which have bluer colours. As potential cluster members we have selected 34 objects (named "BRB") with $J>14.4$ and colours $1.7<I-J<3.8$ (Table 1). We checked that these objects had colours $R-I \gtrsim 1$. Some fainter candidates had unreliable or too faint $R$-band magnitudes and no value is given in Table 1 . We discarded as contaminants the previously identified non-proper motion candidates CFHT-Pl-8 (M 01) and CFHT-PLIZ-25 (M03).

Of the 34 BRB candidates, 22 were identified already by previous surveys (e.g. Béjar 2000, B98; see footnote of Table 1 for all the references). In the magnitude range of the brightest objects we identify an additional candidate, BRB 4, which is also in the 2MASS catalog. This object is not mentioned by B98 and is not in the list of proper motion and $R I$-band photometric candidates from Deacon \& Hambly (2004). The latter survey overlaps the CFHT survey and its $R$-band limiting magnitude is greater than the magnitude of $\mathrm{BRB} 4, R=19.4$. Therefore this object should have been detected. Possibly it was blended or it is not a proper motion cluster member.

The magnitude errors of the BRB candidates are 0.06 mag for $J \sim 14.5-17.0$ and 0.08 mag for $J \sim 17.0-20.0$. Comparing our $J$-band magnitudes with those available in the literature (Martín et al. 2000; Pinfield et al. 2003), i.e. those in the range $J \sim 14.5-17.0$, we find:

1. $\left\langle J_{\mathrm{BRB}}-J_{\text {Martin }}\right\rangle=-0.04 \pm 0.09$, for the objects in common CFHT-Pl-2, 3, 4, 5, 6, 7, 9, 11, 12, 15, 21 and 25. CFHT-Pl-6 and CFHT-Pl-7 present the greatest differences, -0.18 and $-0.26 \mathrm{mag}$, respectively. Both objects lack of $\mathrm{H} \alpha$ emission (Martín et al. 2000), but CFHT-Pl-7 has a proper motion consistent with that of the cluster. In one of our individual $J$-band images, the radial profile of CFHTPl-7 peaks at relatively higher counts and is not well centred, probably due to a cosmic ray; this might explain the greater brightness (it is also supported by the difference with $2 \mathrm{MASS}, J_{\mathrm{BRB}}-J_{2 \mathrm{MASS}}=-0.14$ ). For CFHT-Pl-6, as discussed by M01, if this is an equal mass binary as suggested by its position well above the cluster sequence in the 
Table 1. RIJ photometry and coordinates for the low-mass stars and brown dwarf candidates.

\begin{tabular}{|c|c|c|c|c|c|c|}
\hline Name & Names reported $^{b}$ & $R-I \pm \sigma_{R-I}$ & $I \pm \sigma_{I}$ & $J \pm \sigma_{J}$ & $I-J$ & $\begin{array}{cc}\mathrm{RA}(\mathrm{J} 2000) & \operatorname{Dec}(\mathrm{J} 2000)^{c} \\
(\mathrm{hms}) & \left({ }^{\circ}{ }^{\prime \prime \prime}\right)\end{array}$ \\
\hline BRB 1 & CFHT-P1-1, BPL 242, MBSC 91, (*) & $1.76 \pm 0.13$ & $16.10 \pm 0.07$ & $14.41 \pm 0.03^{d}$ & 1.69 & $035151.6+233450.2$ \\
\hline BRB 2 & CFHT-Pl-2, BPL 267, DH 765, (*) & $2.00 \pm 0.10$ & $16.57 \pm 0.07$ & $14.66 \pm 0.06$ & 1.91 & $035244.3+235415.2$ \\
\hline BRB 3 & CFHT-Pl-6, $(*)$ & $2.38 \pm 0.10$ & $17.13 \pm 0.07$ & $14.71 \pm 0.05$ & 2.41 & $035207.9+235914.6$ \\
\hline BRB 4 & $(*)$ & $2.38 \pm 0.10$ & $17.03 \pm 0.07$ & $14.73 \pm 0.04$ & 2.30 & $034423.2+253844.7$ \\
\hline BRB 5 & CFHT-Pl-3, HHJ 22, BPL 272, MBSC 99, (*) & $1.94 \pm 0.10$ & $16.66 \pm 0.07$ & $14.81 \pm 0.06$ & 1.85 & $035251.8+233348.9$ \\
\hline BRB 6 & CFHT-Pl-4, BPL 280, MBSC 101, (*) & $1.92 \pm 0.10$ & $16.85 \pm 0.07$ & $14.96 \pm 0.06$ & 1.89 & $035309.6+233348.3$ \\
\hline BRB 7 & CFHT-Pl-5, DH 590, (*) & $2.11 \pm 0.10$ & $16.99 \pm 0.07$ & $14.98 \pm 0.06$ & 2.01 & $034844.7+243722.7$ \\
\hline BRB 8 & CFHT-Pl-7, BPL 253, MBSC 108, (*) & $1.84 \pm 0.13$ & $17.48 \pm 0.07$ & $15.16 \pm 0.07$ & 2.32 & $035205.8+241731.7$ \\
\hline BRB 9 & CFHT-Pl-12, BPL 294, CFHT-PLIZ-6, (*) & $2.55 \pm 0.10$ & $18.00 \pm 0.07$ & $15.20 \pm 0.03$ & 2.80 & $035355.1+232337.4$ \\
\hline BRB 10 & CFHT-P1-9, BPL 202, MHOBD 6, (*) & $2.17 \pm 0.10$ & $17.78 \pm 0.07$ & $15.46 \pm 0.07$ & 2.32 & $034915.1+243622.4$ \\
\hline BRB 11 & CFHT-Pl-13, Teide 2, BPL 254, CFHT-PLIZ-3, (*) & $2.11 \pm 0.13$ & $18.14 \pm 0.07$ & $15.50 \pm 0.07$ & 2.64 & $035206.7+241601.4$ \\
\hline BRB 12 & CFHT-Pl-11, Roque 16, BPL 152, (*) & $2.25 \pm 0.10$ & $17.92 \pm 0.07$ & $15.64 \pm 0.07$ & 2.28 & $034739.0+243622.1$ \\
\hline BRB 13 & CFHT-Pl-15, $(*)$ & $2.44 \pm 0.10$ & $18.65 \pm 0.07$ & $15.99 \pm 0.07$ & 2.66 & $035512.5+231738.0$ \\
\hline BRB 14 & CFHT-Pl-21, Calar 3, BPL 235, CFHT-PLIZ-12, (*) & $2.49 \pm 0.11$ & $18.93 \pm 0.07$ & $16.13 \pm 0.05$ & 2.80 & $035125.6+234520.6$ \\
\hline BRB 15 & CFHT-P1-25, BPL 303, CFHT-PLIZ-20, (*) & $2.70 \pm 0.13$ & $19.68 \pm 0.07$ & $16.71 \pm 0.04$ & 2.98 & $035405.3+233400.2$ \\
\hline BRB 16 & PIZ 1 & - & $20.10 \pm 0.12^{e}$ & $16.84 \pm 0.07$ & 3.26 & $034831.4+243437.7$ \\
\hline $\mathrm{BRB} 17^{a}$ & $(* *)$ & - & $20.92 \pm 0.07$ & $17.42 \pm 0.06$ & 3.50 & $035408.31+235433.4$ \\
\hline $\mathrm{BRB} 18^{a}$ & CFHT-PLIZ-28, $(* *)$ & $2.37 \pm 0.17$ & $21.20 \pm 0.07$ & $17.61 \pm 0.07$ & 3.59 & $035414.08+231752.2$ \\
\hline $\mathrm{BRB} 19^{a}$ & - & $2.25 \pm 0.14$ & $20.95 \pm 0.09$ & $17.79 \pm 0.05$ & 3.16 & $035451.49+234512.2$ \\
\hline $\mathrm{BRB} 20^{a}$ & CFHT-PLIZ-35, $(*),(* *)$ & $2.55 \pm 0.23$ & $21.47 \pm 0.06$ & $18.06 \pm 0.07$ & 3.40 & $035239.16+244629.7$ \\
\hline $\mathrm{BRB} 21^{a}$ & $(* *)$ & $2.34 \pm 0.21$ & $21.68 \pm 0.08$ & $18.14 \pm 0.05$ & 3.54 & $035410.27+234140.3$ \\
\hline $\mathrm{BRB} 22^{a}$ & CFHT-PLIZ 2141 & $2.14 \pm 0.20$ & $21.97 \pm 0.08$ & $18.31 \pm 0.05$ & 3.65 & $034431.27+253515.1$ \\
\hline $\mathrm{BRB} 23^{a}$ & $(* *)$ & $2.13 \pm 0.27$ & $22.03 \pm 0.10$ & $18.55 \pm 0.10$ & 3.49 & $035039.53+250254.5$ \\
\hline BRB 24 & $(*)$ & $2.95 \pm 0.51$ & $22.01 \pm 0.09$ & $18.71 \pm 0.08$ & 3.30 & $034819.65+245209.5$ \\
\hline BRB 25 & - & - & $22.24 \pm 0.09$ & $18.74 \pm 0.07$ & 3.50 & $035259.73+235156.0$ \\
\hline BRB 26 & - & $1.93 \pm 0.30$ & $22.07 \pm 0.08$ & $18.76 \pm 0.10$ & 3.31 & $034856.17+250943.1$ \\
\hline $\mathrm{BRB} 27^{a}$ & CFHT-PLIZ 1262, (**) & $2.30 \pm 0.32$ & $22.64 \pm 0.11$ & $18.89 \pm 0.09$ & 3.74 & $034427.24+254441.9$ \\
\hline $\mathrm{BRB} 28^{a}$ & $(*),(* *)$ & $2.64 \pm 0.45$ & $22.36 \pm 0.08$ & $19.02 \pm 0.10$ & 3.33 & $035254.92+243718.6$ \\
\hline $\mathrm{BRB} 29^{a}$ & - & - & $22.84 \pm 0.10$ & $19.05 \pm 0.07$ & 3.79 & $035401.43+234958.1$ \\
\hline BRB 30 & - & $1.98 \pm 0.23$ & $22.48 \pm 0.10$ & $19.17 \pm 0.07$ & 3.31 & $034756.96+242858.4$ \\
\hline BRB 31 & - & $2.50 \pm 0.25$ & $23.05 \pm 0.10$ & $19.29 \pm 0.10$ & 3.76 & $035125.93+241532.2$ \\
\hline BRB 32 & - & - & $22.72 \pm 0.12$ & $19.36 \pm 0.09$ & 3.36 & $034900.86+244138.5$ \\
\hline $\mathrm{BRB} 33^{a}$ & - & - & $23.31 \pm 0.20$ & $19.63 \pm 0.09$ & 3.68 & $035120.15+234518.4$ \\
\hline BRB 34 & - & - & $23.01 \pm 0.16$ & $19.64 \pm 0.09$ & 3.37 & $034846.55+244503.2$ \\
\hline
\end{tabular}

$a$ With follow-up observation for proper motion measurement (see Table 2).

${ }^{b}$ (*) (Béjar 2000), CFHT-Pl (B98), BPL (Pinfield et al. 2000), MBSC and CFHT-PLIZ (M03), (**) (Bihain et al. 2005), DH (Deacon \& Hambly 2004), CFHT-PLIZ (Bouy et al. 2006), HHJ 22 (Hambly et al. 1993), MHOBD 6 (Stauffer et al. 1998a), Teide 2 (Martín et al. 1998a), Roque 16 (Zapatero Osorio et al. 1997b), Calar 3 (Martin et al. 1996), PIZ 1 (Cossburn et al. 1997). In Fig. 1 we overplotted the 14 CFHT-Pl objects (open circles) and the 20 other objects (filled circles).

${ }^{c}$ Coordinates of CFHT-Pl, CFHT-PLIZ, PIZ 1 and the other objects are from B98, M03, Cossburn et al. (1997) and the present study, respectively.

${ }^{d} J$-band estimate from Martín et al. (2000) because our estimate is bad pixel-contaminated.

${ }^{e} I$-band estimate corrected for a $\sim 0.25 \mathrm{mag}$ reddening due to a close star and ray light traces; the offset was obtained by comparing to objects of similar counts in clean sky areas of the image. Converting the Kitt Peak filter measurement $I_{K P}=19.64$ from Cossburn et al. (1997) to Cousins with the transform equation given by Jameson et al. (2002), we find $I=19.9$.

colour-magnitude diagram (B98; see also Fig. 2), the binarity might affect the determination of its short term proper motion and therefore explain its deviance from the cluster peculiar motion. The binarity could also explain some of the magnitude difference observed.

2. $\left\langle J_{\mathrm{BRB}}-J_{\text {Pinfield }}\right\rangle=0.04 \pm 0.09$, for CFHT-Pl-2, 3, 4, 7, 9, 11, 12, 13, 21 and 25. CFHT-Pl-4 and CFHT-Pl-11 present the greatest differences, $0.19 \mathrm{mag}$ and $0.15 \mathrm{mag}$, respectively. These are also found for 2MASS: $J_{\mathrm{BRB}}-J_{2 \mathrm{MASS}}=0.11$ and 0.19, respectively. But CFHT-Pl-4 and CFHT-Pl-11 have no deviant proper motions or anomalous positions in the colour-magnitude diagram.

Our I-band magnitudes rely on an approximate offset to the magnitudes from B98 and have an error of $\sim 0.1 \mathrm{mag}$. The I-band magnitude from B98 is obtained after transformation onto the standard Cousins system, with observation of red Landolt standards. Due to the lack of very red standard stars, the transformation is linearly extrapolated for the red and faint objects and could produce systematic errors. Comparing our magnitudes to those in the Cousins system from M03, for the candidates in common and 1-2 mag fainter than CFHT-Pl-25 (one of the faintest objects from B98), we observe a difference $I_{\mathrm{BRB}}-I_{\text {Moraux }} \sim 0.1$, which is quite small in comparison with the $I-J>3.3$ threshold that we applied to select the faint candidates.

Finally, from the 34 BRB Pleiades candidates, the 18 faintest have $J \gtrsim 17$ and $I-J \gtrsim 3.3$, the magnitudes and colours expected for L-type dwarfs.

\subsection{Follow-up observations}

\subsection{1. $K_{\mathrm{s}}$-band imaging}

Follow-up $K_{\mathrm{s}}$-band imaging with CAIN-II at the $1.55 \mathrm{~m}$ Telescopio Carlos Sánchez (TCS; Teide Observatory, Tenerife) 
Table 2. $H K_{\mathrm{s}}$-band follow-up observations.

\begin{tabular}{|c|c|c|c|c|}
\hline Telescope/instrument & Filter(s) & Date & Object(s) & $\langle c\rangle,\langle l\rangle^{a}$ \\
\hline TCS/CAIN - II & $K_{\mathrm{s}}$ & 2004 Dec. 7 & BRB $17,18,21,23,26$ & $\sim 17.6, \sim 18.2$ \\
\hline TCS/CAIN - II & $K_{\mathrm{s}}$ & 2004 Dec. 8 & BRB 20,28 & $\sim 18.1, \sim 18.6$ \\
\hline WHT/LIRIS & $H$ & 2005 Jan. 23 & BRB 17,23 & $\sim 19.5, \sim 20.0$ \\
\hline WHT/LIRIS & $H$ & 2005 Jan. 24 & BRB 20, 28 & $\sim 19.0, \sim 19.5$ \\
\hline $3.5 \mathrm{~m} \mathrm{Calar} / \Omega 2000$ & $H$ & 2005 Feb. 1 & BRB $17,18,21$, Teide 1 & $\sim 18.5, \sim 19.3$ \\
\hline TCS/CAIN - II & $K_{\mathrm{s}}$ & 2005 Mar. 6 & BRB 25 & $\sim 16.5, \sim 17.0$ \\
\hline WHT/LIRIS & $K_{\mathrm{s}}$ & 2005 Mar. 25 & BRB 27, Teide 1 & $\sim 18.8, \sim 19.3$ \\
\hline TCS/CAIN - II & $K_{\mathrm{s}}$ & 2005 Oct. 17 & BRB 19 & $\sim 17.5, \sim 18.5$ \\
\hline TCS/CAIN - II & $K_{\mathrm{s}}$ & 2005 Oct. 18 & BRB 22 & $\sim 18.0, \sim 19.0$ \\
\hline $3.5 \mathrm{~m} \mathrm{Calar} / \Omega 2000$ & $H$ & 2005 Oct. 21 & BRB 19 & $\sim 19.0, \sim 19.5$ \\
\hline TCS/CAIN - II & $K_{\mathrm{s}}$ & 2005 Oct. 21 & BRB 24, 34 & $\sim 17.8, \sim 18.8$ \\
\hline $3.5 \mathrm{~m} \mathrm{Calar} / \Omega 2000$ & $H, K_{\mathrm{s}}$ & 2005 Oct. 22 & BRB 22 & $\sim 19.0, \sim 19.5$ \\
\hline TCS/CAIN - II & $K_{\mathrm{s}}$ & 2005 Oct. 22 & BRB 29 & $\sim 18.0, \sim 19.0$ \\
\hline $3.5 \mathrm{~m} \mathrm{Calar} / \Omega 2000$ & $H$ & 2005 Oct. 23 & BRB 29 & $\sim 18.5, \sim 19.0$ \\
\hline TCS/CAIN - II & $K_{\mathrm{s}}$ & 2005 Oct. 23 & BRB 31, 33 & $\sim 18.8, \sim 19.5$ \\
\hline $3.5 \mathrm{~m} \mathrm{Calar} / \Omega 2000$ & $K_{\mathrm{s}}$ & 2005 Oct. 24 & BRB 33 & $\sim 19.0, \sim 19.5$ \\
\hline $3.5 \mathrm{~m} \mathrm{Calar} / \Omega 2000$ & $H$ & 2005 Oct. 25 & BRB 33 & $\sim 19.0, \sim 19.5$ \\
\hline TCS/CAIN - II & $K_{\mathrm{s}}$ & 2005 Oct. 28 & BRB 30, 32 & $\sim 18.0, \sim 19.0$ \\
\hline
\end{tabular}

${ }^{a}$ Average completeness and limiting magnitudes.

was performed for all $18 I J$-band photometric substellar candidates (see the observation log in Table 2). The CAIN-II camera has a $256 \times 256 \mathrm{HgCdTe}$ Nicmos 3 detector with a pixel scale of $1.00^{\prime \prime}$ pixel $^{-1}$ in wide field configuration, providing a $4.2^{\prime} \times 4.2^{\prime}$ field of view. The observation macro consisted in $6 \mathrm{~s}$ exposures $\times 10$ repetitions $\times 10$ dither positions. Total exposure times spanned a range of 10-90 min, depending on the expected brightness of the candidates and the seeing. Data were skysubtracted, flat-fielded (using sky flats instead of dome flats), aligned and combined in a similar way as for the $J$-band data (Sect. 2.1). We also performed the photometry and estimated the completeness and limiting magnitudes as described for the $J$-band. On average, about six 2MASS calibrators were used per field. Average completeness and limiting magnitudes during the different nights are indicated in Table 2.

\subsection{2. $H K_{\mathrm{s}}$-band imaging for astrometry}

For the proper motion determination of the candidates, we obtained subarcsecond $H$ - and $K_{\mathrm{s}}$-band images with the Longslit Intermediate Resolution Infrared Spectrograph (LIRIS) at the $4.2 \mathrm{~m}$ William Hershel Telescope (WHT; Roque de los Muchachos Observatory, La Palma) and $H$-band images with $\Omega 2000$ at the $3.5 \mathrm{~m}$ Telescope of CAHA (see Table 2).

We decided also to observe the Pleiades brown dwarf Teide 1 (Rebolo et al. 1995) with both LIRIS and $\Omega 2000$ to improve its proper motion measurement and to check if there are any systematic errors in the proper motion measurements using different instruments. Our first epoch image was a $12^{\prime} \times 12^{\prime}$ reduced image, obtained with the TEK3 CCD detector $\left(0.7^{\prime \prime}\right.$ pixel $\left.^{-1}\right)$ at the prime focus of the $2.5 \mathrm{~m}$ Isaac Newton Telescope (INT; Roque de los Muchachos Observatory, La Palma) on 1995 December 19 (Zapatero Osorio 2005, private communication).

The LIRIS camera has a $1024 \times 1024 \mathrm{HgCdTe}$ Hawaii detector with a pixel scale of $0.25^{\prime \prime}$ pixel $^{-1}$ and a $4.2^{\prime} \times 4.2^{\prime}$ field of view, whereas the $\Omega 2000$ camera has a $2048 \times 2048 \mathrm{HgCdTe}$ Hawaii 2 detector with a pixel scale of $0.45^{\prime \prime}$ pixel $^{-1}$ and a field of view of $15.4^{\prime} \times 15.4^{\prime}$. The observation macros consisted of: $5 \mathrm{~s}$ exposures $\times 18$ repetitions $\times 5$ dithers (LIRIS night
2005 January 23), 5 s exposures $\times 12$ repetitions $\times 5$ dithers (LIRIS; January 24), $3 \mathrm{~s} \times 20$ coadds $\times 7$ dithers $(\Omega 2000$; February 1), $20 \mathrm{~s} \times 6$ repetitions $\times 5$ dithers (LIRIS; March 25), $2 \mathrm{~s} \times 20$ coadds $\times 15$ dithers $(\Omega 2000$; October 21,22 and 23$)$, $2 \mathrm{~s} \times 15$ coadds $\times 20$ dithers $(\Omega 2000$; October 24$)$ and $3 \mathrm{~s} \times$ 15 coadds $\times 20$ dithers $(\Omega 2000$; October 25$)$. The total exposure times were chosen to achieve enough $\mathrm{S} / \mathrm{N}(\gtrsim 20$ in the peak) for precise measurement of the position of the candidates. They ranged between 5 and $40 \mathrm{~min}$, depending on the expected brightness of the candidates and the seeing.

The raw images were first bad-pixel corrected using a badpixel mask and the IRAF routine PROTO.FIXPIX. The mask was obtained from the flat (sky-flat for LIRIS and dome-flat for $\Omega 2000$ ) using NOAO . IMRED . CCDRED . CCDMASK. The images were then sky-subtracted - sky images were obtained by combining 5-20 consecutive images of similar sky counts - and divided by the flat.

The resulting images were distortion corrected, aligned using 10-20 reference stars and combined. For LIRIS these last three steps were performed with the LDEDITHER task within the package IRAF.LIRIS. LIMAGE developed by J. A. Acosta-Pulido. For $\Omega 2000$, the images were distortion corrected by projecting them on the celestial coordinate grid with MSCIMAGE. The grid was obtained by astrometry with the USNO-A2 catalog and the script myasrtrom.cl from E. Puddu. This script invokes CCFIND, that correlates the catalog sources with those in the image, and CCMAP, which computes the plate solution. Usually, 50-100 stars were accepted by a Legendre order- 4 transformation fit and a rejection threshold of 1.5 sigma, providing a projection transformation with standard deviation $\sim 0.12^{\prime \prime}$ in both right ascension and declination. The distortion corrected images of $\Omega 2000$ were then aligned and combined using the INTALIGN task within the LIRIS package.

The $H K_{\mathrm{s}}$-band photometry was obtained similarly as for the $J$-band data. On average, six and eleven 2MASS calibrators were used per LIRIS and $\Omega 2000$ field, respectively. The average completeness and limiting magnitudes during the different nights are indicated in Table 2. 
Table 3. Follow-up $H K_{\mathrm{s}}$ photometry of the low-mass brown dwarf candidates.

\begin{tabular}{|c|c|c|c|c|c|c|}
\hline Name & $J$ & $I-J \pm \sigma_{I-J}$ & $H \pm \sigma_{H}$ & $\overline{J-H \pm \sigma_{J-H}}$ & $K_{\mathrm{s}} \pm \sigma_{K_{\mathrm{s}}}$ & $\bar{J} J-K_{\mathrm{s}} \pm \sigma_{J-K_{\mathrm{s}}}$ \\
\hline BRB $17^{a}$ & 17.42 & $3.50 \pm 0.09$ & $16.75 \pm 0.02$ & $0.67 \pm 0.06$ & $16.20 \pm 0.06$ & $1.22 \pm 0.08$ \\
\hline BRB $18^{a}$ & 17.61 & $3.59 \pm 0.10$ & $16.74 \pm 0.06$ & $0.87 \pm 0.09$ & $16.08 \pm 0.07$ & $1.53 \pm 0.09$ \\
\hline $\mathrm{BRB} 19^{a}$ & 17.79 & $3.16 \pm 0.10$ & $16.99 \pm 0.05$ & $0.80 \pm 0.07$ & $16.67 \pm 0.05$ & $1.12 \pm 0.07$ \\
\hline $\mathrm{BRB} 20^{a}$ & 18.06 & $3.40 \pm 0.09$ & $17.33 \pm 0.05$ & $0.73 \pm 0.08$ & $16.56 \pm 0.07$ & $1.50 \pm 0.10$ \\
\hline BRB $21^{a}$ & 18.14 & $3.54 \pm 0.09$ & $17.05 \pm 0.07$ & $1.09 \pm 0.09$ & $16.39 \pm 0.07$ & $1.75 \pm 0.09$ \\
\hline $\mathrm{BRB} 22^{a}$ & 18.31 & $3.65 \pm 0.09$ & $17.38 \pm 0.04$ & $0.94 \pm 0.06$ & $16.69 \pm 0.04$ & $1.62 \pm 0.06$ \\
\hline $\mathrm{BRB} 23^{a}$ & 18.55 & $3.49 \pm 0.14$ & $17.47 \pm 0.06$ & $1.08 \pm 0.11$ & $16.65 \pm 0.06$ & $1.90 \pm 0.11$ \\
\hline BRB 24 & 18.71 & $3.30 \pm 0.12$ & - & - & $17.49 \pm 0.07$ & $1.22 \pm 0.11$ \\
\hline BRB 25 & 18.74 & $3.50 \pm 0.12$ & - & - & $16.91 \pm 0.24$ & $1.83 \pm 0.25$ \\
\hline BRB 26 & 18.76 & $3.31 \pm 0.13$ & - & - & $17.81 \pm 0.10$ & $0.95 \pm 0.14$ \\
\hline $\mathrm{BRB} 27^{a}$ & 18.89 & $3.74 \pm 0.14$ & - & - & $17.16 \pm 0.09$ & $1.74 \pm 0.12$ \\
\hline $\mathrm{BRB} 28^{a}$ & 19.02 & $3.33 \pm 0.12$ & $17.89 \pm 0.05$ & $1.13 \pm 0.11$ & $17.00 \pm 0.08$ & $2.02 \pm 0.13$ \\
\hline $\mathrm{BRB} 29^{a}$ & 19.05 & $3.79 \pm 0.12$ & $17.76 \pm 0.05$ & $1.29 \pm 0.09$ & $16.88 \pm 0.08$ & $2.17 \pm 0.11$ \\
\hline BRB 30 & 19.17 & $3.31 \pm 0.12$ & - & - & $17.65 \pm 0.09$ & $1.52 \pm 0.11$ \\
\hline BRB 31 & 19.29 & $3.76 \pm 0.14$ & - & - & $18.20 \pm 0.15$ & $1.09 \pm 0.18$ \\
\hline BRB 32 & 19.36 & $3.36 \pm 0.15$ & - & - & $17.42 \pm 0.20$ & $1.94 \pm 0.22$ \\
\hline BRB $33^{a}$ & 19.63 & $3.68 \pm 0.22$ & $18.71 \pm 0.07$ & $0.92 \pm 0.11$ & $17.87 \pm 0.06$ & $1.76 \pm 0.11$ \\
\hline BRB 34 & 19.64 & $3.37 \pm 0.18$ & - & - & $18.8 \pm 0.5$ & $0.9 \pm 0.5$ \\
\hline
\end{tabular}

${ }^{a}$ With proper motion measurement.

\subsection{Proper motion analysis}

The proper motion measurements were obtained as follows. First we selected non-saturated and well defined $(S / N>10)$ single objects among all those that appeared within $\sim 3^{\prime}$ of a brown dwarf candidate. We measured their centres in the first- and the second-epoch images with the IRAF task CENTER (DIGIPHOT.DAOPHOT package), with a precision of 0.01-0.1 pixels in both image dimensions. For BRB 22 and 33 we used the $H$-band $\Omega 2000$ images as second-epoch images, because they provided greater $S / N$ for the candidates than the $\Omega 2000 K_{\mathrm{s}}$-band images. Then we computed the transformation from second-epoch image to first-epoch image with GEOMAP. For the $\Omega 2000$ image of Teide 1, we performed a transformation from first- to second-epoch (i.e. from TEK3 to $\Omega 2000$ ), because the destination image had a wider field and a smaller pixel scale and therefore provided more accurate equatorial astrometry with the USNO-A2 catalog. With GEOMAP we selected the objects whose positions minimized the transformation error and could thus be considered as reference objects. A Legendre function of order 3 was used when more than 12 objects were available, and of order 2 for fewer objects. The error, related to the scatter of these objects, ranged over $0.03-0.15$ pixels. For the candidate BRB 23 the error in the $y$ dimension was as high as 0.6 pixels, because of vertical smearing affecting the object positions in the $I$-band image (field CFHT 6). With GEOXYTRAN we used the transformation to predict the candidate position $\left(x_{1 p}, y_{1 p}\right)$ in a first-epoch image, and compared it with the measured one, $\left(x_{1}, y_{1}\right)$, obtaining the pixel shifts $\left(\Delta_{x_{1}}, \Delta_{y_{1}}\right)=\left(x_{1 p}-x_{1}, y_{1 p}-y_{1}\right)$. The plate solution of a first-epoch (or destination) image was obtained with the USNO-A2 catalog and the myasrtrom.cl script, in a similar way as explained above. Then using WCSCTRAN we converted the shifts in pixels to shifts in equatorial coordinates. Due to the precision of the equatorial astrometry $\left(\sigma \sim 0.12^{\prime \prime}\right)$, its error contribution to the proper motion was negligible. But for the LIRIS proper motion measurement of Teide 1, the best first epoch TEK3 fit had a relatively high standard deviation $\left(\sigma_{\mathrm{RA}}=0.6^{\prime \prime}, \sigma_{\mathrm{DEC}}=0.7^{\prime \prime}\right)$ and the corresponding proper motion value differed by almost $\sim 1$ mas $\mathrm{yr}^{-1}$ with those derived from less precise fits; thus we added $\sim 1$ mas $\mathrm{yr}^{-1}$ to the proper motion error in this case. In general, we assumed that the proper motion error was:

$$
\begin{aligned}
& \left(\sigma_{\mu_{\alpha} \cos \delta}, \sigma_{\mu_{\delta}}\right)= \\
& \left(\frac{f_{\mu_{\alpha} \cos \delta}\left(\sigma_{x 1}^{2}+\sigma_{x 2}^{2}+\sigma_{t x}^{2}\right)}{\Delta t}, \frac{f_{\mu_{\delta}}\left(\sigma_{y 1}^{2}+\sigma_{y 2}^{2}+\sigma_{t y}^{2}\right)}{\Delta t}\right),
\end{aligned}
$$

where $\sigma_{x 1, y 1}, x 2, y 2$ are the errors of the $(x, y)$ pixel positions in the first- (1) and second-epoch (2) images, $\sigma_{t x, t y}$ the error of the transformation between these images, $f_{\mu_{\alpha} \cos \delta, \mu_{\delta}}$ the transformation from $(x, y)$ to (RA, DEC) coordinates, and $\Delta t$ the time in Julian years between the two epochs of observation.

\section{Proper motion Pleiades low-mass brown dwarfs}

\subsection{Photometry and proper motion results}

The $H K_{\mathrm{s}}$ magnitudes for the red and faint brown dwarf candidates that we have followed-up are given in Table 3 . The $H$-band magnitude of BRB 17 is the error-weighted average from the values obtained with $\Omega 2000$ and LIRIS, and the $K_{\mathrm{s}}$-band magnitudes of BRB 22 and 33 are the error-weighted averages from the values obtained with $\Omega 2000$ and TCS. Our 18 follow-up objects have magnitudes $20.9<I<23.3,17.4<J<19.6$, and colours $3.2<I-J<3.8,0.7<J-H<1.3$ and $0.9<J-K_{\mathrm{s}}<2.2$. Most of them remain good $\mathrm{L}$ dwarf candidates, what supports our $I-J$ selection criterion. But BRB 24, 26, 30, 31 and 34 present much bluer $J-K_{\mathrm{s}}$ colours than cluster proper motion members with similar $J$-band magnitude (see Fig. 5). Moreover, if they were late $\mathrm{L}$ or $\mathrm{T}$ dwarfs, they would certainly present much redder $I-J$ colours (>4-5 mag). We will assume that these are non-cluster members. Finally BRB 32, with its red $J-K_{\mathrm{s}}$ and relatively blue $I-J$ colours, remains a possible cluster member.

The proper motion results obtained for 11 of the brown dwarf candidates and two lithium brown dwarfs (Teide 1 and Calar 3) are listed in Table 4 and are represented by small circles in the vector point diagram of Fig. 3. Because Calar 3 (Martin et al. 1996; Rebolo et al. 1996) is at a few arcminutes from BRB 33, we could also measure its proper motion and found a very good agreement with the measurement of M01. We note that the 
Table 4. Proper motions of the low-mass brown dwarf candidates and the lithium brown dwarfs Teide 1 and Calar 3.

\begin{tabular}{cccc}
\hline \hline Name & Instrument & $\begin{array}{c}\mu_{\alpha} \cos \delta \pm \sigma_{\mu_{\alpha} \cos \delta} \\
\left(\mathrm{mas} \mathrm{yr}^{-1}\right)\end{array}$ & $\begin{array}{c}\mu_{\delta} \pm \sigma_{\mu_{\delta}} \\
\left(\mathrm{mas} \mathrm{yr}^{-1}\right)\end{array}$ \\
\hline Teide 1 & LIRIS & $22.5 \pm 3.6$ & $-40.5 \pm 4.0$ \\
Teide 1 & $\Omega 2000$ & $19.2 \pm 2.5$ & $-45.5 \pm 3.4$ \\
Calar 3 & $\Omega 2000$ & $24.5 \pm 2.2$ & $-32.6 \pm 2.2$ \\
\hline BRB 17 & LIRIS & $20.9 \pm 1.5$ & $-22.8 \pm 2.4$ \\
BRB 17 & $\Omega 2000$ & $17.7 \pm 2.9$ & $-20.7 \pm 3.1$ \\
BRB 18 & $\Omega 2000$ & $28.4 \pm 2.7$ & $-37.4 \pm 2.7$ \\
BRB 19 & $\Omega 2000$ & $75.7 \pm 1.0$ & $-143.9 \pm 7.7$ \\
BRB 20 & LIRIS & $21.8 \pm 3.0$ & $-48.1 \pm 2.0$ \\
BRB 21 & $\Omega 2000$ & $18.1 \pm 3.4$ & $-49.1 \pm 4.1$ \\
BRB 22 & S2000 & $18.8 \pm 3.5$ & $-51.9 \pm 2.9$ \\
BRB 23 & LIRIS & $29.2 \pm 3.0$ & $-25.6 \pm 14.7$ \\
BRB 27 & LIRIS & $35.3 \pm 3.7$ & $-48.8 \pm 2.0$ \\
BRB 28 & LIRIS & $15.1 \pm 3.1$ & $-37.4 \pm 2.1$ \\
BRB 29 & S2000 & $26.4 \pm 3.3$ & $-48.8 \pm 2.9$ \\
BRB 33 & $\Omega 2000$ & $-4.1 \pm 3.0$ & $-5.2 \pm 3.7$ \\
\hline
\end{tabular}

double-check measurements of Teide 1 and BRB 17 are fully consistent within the statistic errors. In Fig. 3 are represented also: $(i)$ the reference objects used to estimate the proper motions (asterisks), (ii) the circle of radius three times the greatest component of the average proper motion error derived from Table $4\left(\left(\left\langle\sigma_{\mu_{\alpha} \cos \delta}\right\rangle,\left\langle\sigma_{\mu_{\delta}}\right\rangle\right)=(3.0,4.3)\right.$ mas $\left.\mathrm{yr}^{-1}\right)$. This circle is centred on the cluster average proper motion, $\left(\mu_{\alpha} \cos \delta, \mu_{\delta}\right)=$ $(19.15 \pm 0.23,-45.72 \pm 0.18)$ mas $\mathrm{yr}^{-1}$ (Robichon et al. 1999), and we will assume that the objects within are cluster members.

The L dwarf candidates BRB 17, 23 and 27 lie outside the circle in Fig. 3 and their membership is considered uncertain. The proper motion dispersion of the seven objects within this circle is $(5.2,5.9)$ mas $\mathrm{yr}^{-1}$. Subtracted quadratically by the average proper motion error, it yields $(4.3,4.1){\mathrm{mas} \mathrm{yr}^{-1} \text {, as an estimate }}^{2}$ of the intrinsic velocity dispersion of Pleiades brown dwarfs. This velocity dispersion is at least four times that of Pleiades stars with masses $\gtrsim 1 M_{\odot}$ (Jones 1970; Pinfield et al. 1998) and appears consistent with the linear relationship of equipartition of energy, between the velocity dispersion and the inverse square root of the mass for cluster members, as expected in an nearly relaxed cluster (Pinfield et al. 1998 and Fig. 4 therein).

We cannot rule out that some of the $I J$ candidates located close to the region surrounding the circle in Fig. 3 are in fact cluster members (although field contaminants are neither excluded, see Sect. 4.1 and Table 5). This is actually the case of the Pleiades lithium brown dwarf Calar 3, suggesting that the intrinsic velocity dispersion of brown dwarfs could be even greater than our previous estimate. We need further data (for instance from lithium observations) to study the membership of the peculiar $I J$ candidates. Note also the case of CFHT-PI-15 (B98), which has a proper motion of $(66.5 \pm 8.1,-54.1 \pm 8.1) \mathrm{mas} \mathrm{yr}^{-1}$ (M 01), thus located far from the cluster point in the proper motion diagram, although it presents a radial velocity consistent with that of the cluster, $\mathrm{Li}$ absorption, $\mathrm{H}_{\alpha}$ emission and a red $I-K_{\mathrm{s}}$ colour (Stauffer et al. 1998b; Martín et al. 2000).

However, BRB 19 and BRB 33 appear clearly to be nonproper motion members; BRB 19 has an especially high proper motion. Finally, our error-weighted average estimate for Teide 1 is $(20.3 \pm 2.1,-43.4 \pm 2.6)$ mas $\mathrm{yr}^{-1}$, very close to the cluster proper motion. It also agrees with the measurement obtained

2 At the cluster distance, it corresponds to a tangential velocity of $\sim 2.5 \mathrm{~km} \mathrm{~s}^{-1}$.

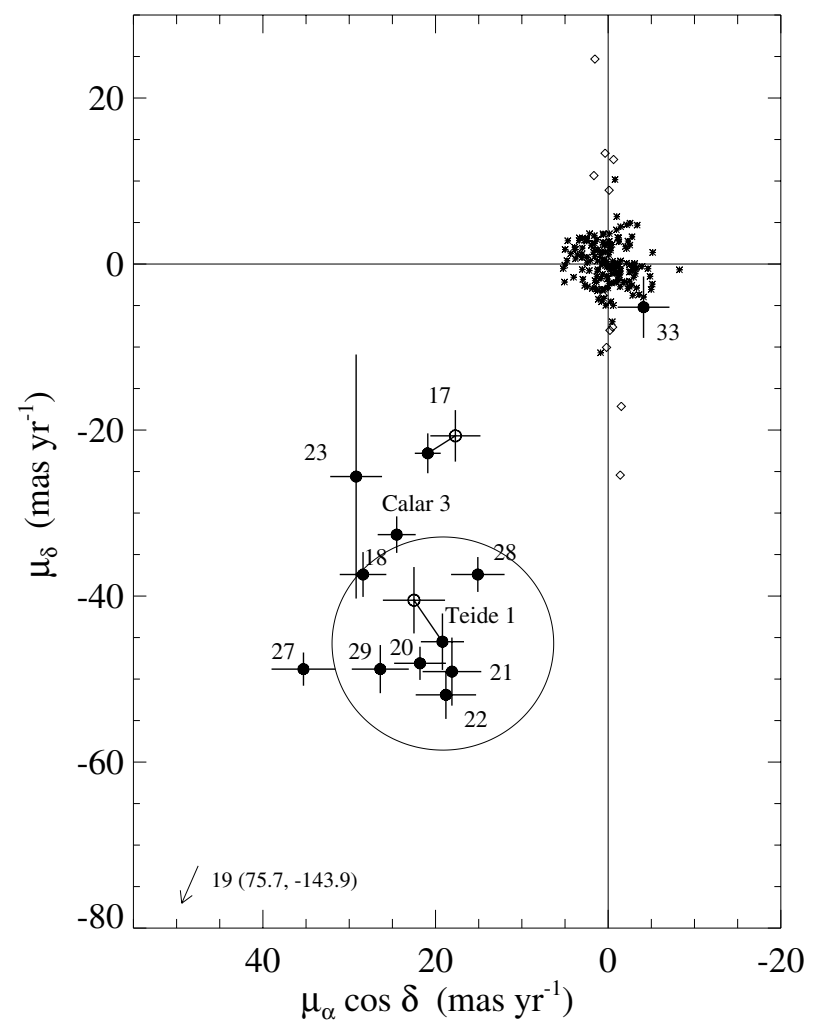

Fig. 3. Vector point diagram of proper motion for the candidates (small circles with BRB number indicated) and the reference objects (asterisks). The results for the Pleiades lithium brown dwarfs Teide 1 and Calar 3 are also shown. Double-check objects are linked by a solid line. The plotted large circle is centred on the Pleiades average proper motion (see text for details). The reference objects of BRB 23 (small squares) present a large dispersion in declination which causes the large error bar of this candidate. The arrow plotted for BRB 19 indicates a location outside the diagram.

by Rebolo et al. (1995), the only previous estimate $(16 \pm 9$, $-35 \pm 16)$ mas $\mathrm{yr}^{-1}$, but with large conservative errors. These measurements, together with the clear lithium detection (Rebolo et al. 1996), thus fully confirm Teide 1 as a Pleiades brown dwarf. Also, the photometry obtained with $\Omega 2000$ and LIRIS, $H=15.54 \pm 0.06$ and $K_{\mathrm{s}}=15.07 \pm 0.09$, respectively, agrees with that of Jameson et al. (2002), $H=15.65 \pm 0.09$ and $K=15.08 \pm 0.05$.

\subsection{Pleiades $L d$ warf sequence}

In the $J$ versus $I-J$ colour-magnitude diagram (Fig. 2), the six cluster proper motion members (large filled circles) define the Pleiades L dwarf sequence. Note that the three possible members with relatively high proper motion (circled filled circles) share the same sequence (as well as in the near-IR colour-magnitude diagrams, Figs. 4 and 5).

In Fig. 2 we also present theoretical predictions for the $I$ and $J$ bandpasses. The solid line corresponds to the DUSTY isochrone and the dash-dotted line corresponds to the NextGen isochrone from Baraffe et al. (1998) for an age 125 Myr. Masses in solar units are shown for the first isochrone. The NextGen model provides a good fit for $J<16.0-16.5$ and the DUSTY model provides a good fit for $J \lesssim 18$. Both models fail to reproduce the photometric sequence at fainter magnitudes; the former does not account for the onset of dust formation in cooler atmospheres, whereas the latter overestimates the $I-J$ colour. 


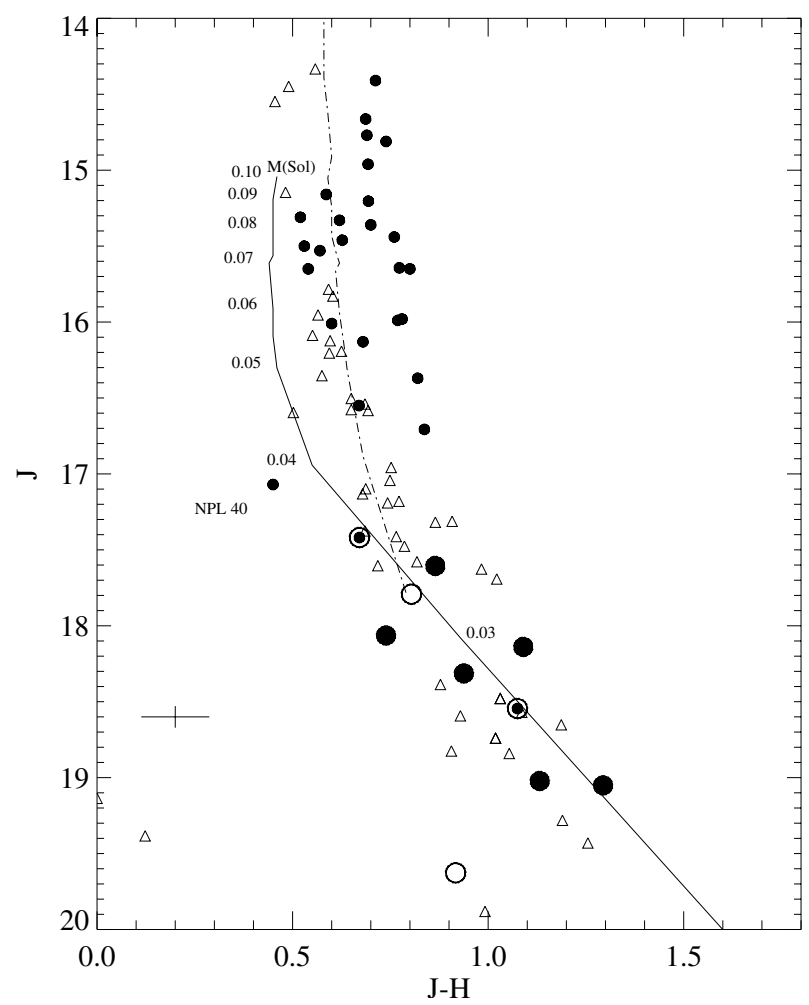

Fig. 4. $J$ versus $J-H$ colour-magnitude diagram for all the Pleiades very low-mass star and brown dwarf candidates with lithium or proper motion consistent with membership (filled circles). Their photometry was compiled from the present study, Martín et al. (2000), the 2MASS catalog, Zapatero Osorio et al. (1997b), Béjar (2000) and Jameson et al. (2002). The circular symbols $(J>17.4)$, lines and triangles are defined as in Fig. 2. The typical error bars for the probable and possible cluster members by proper motion are represented on the left.

Also in Fig. 2 we show the sequence of field dwarfs (triangles) with parallactic distances, translated to the distance of the Pleiades cluster. These nearby cool dwarfs are compiled from the literature by Caballero (2006). Their $I$ - and $J H K_{\mathrm{s}}$-band photometry are from Dahn et al. $(2000,2002)$ and the 2MASS Point Source Catalog, respectively. For the $\mathrm{M}$ dwarfs, the parallaxes are from Dahn et al. $(2000,2002)$ and the spectral types are from Golimowski et al. (2004), Leggett et al. (2000, 2002) and Dahn et al. (2002). For the $\mathrm{L}$ and $\mathrm{T}$ dwarfs, the parallaxes are from Perryman et al. (1997), Dahn et al. (2002) and Vrba et al. (2004), and the spectral types are mostly from Vrba et al. (2004), else from Leggett et al. (2002) and Geballe et al. (2001). In Fig. 2 the spectral types range over $\sim \mathrm{M} 3-\mathrm{T} 2$ from top to bottom. At magnitudes $J<17$ the field dwarfs are bluer than the cluster brown dwarfs, but at $J>17$ the field sequence begins to intercept that of the cluster and to follow it slightly towards redder colours.

In the $J$ versus $J-H$ and $J-K$ diagrams (Figs. 4 and 5), we show the Pleiades very low-mass stars and brown dwarf candidates having lithium in their atmospheres or proper motions consistent with membership in the cluster (filled circles). The circular symbols $(J>17.4)$, lines and triangles are defined as in Fig. 2. The DUSTY and NextGen isochrones provide relatively good colour predictions for $J>17$ and $J<17$, respectively, in both diagrams. The fact that the DUSTY model agrees with the observations in the $J$ versus $J-H$ and $J-K$ diagrams but not in the $J$ versus $I-J$ diagram supports the possible underestimation of the far red flux ( $I$ band) relative to the near-IR flux ( $J$-, $H$ - and $K$ bands). In Fig. 4 , the M9 Pleiades brown dwarf

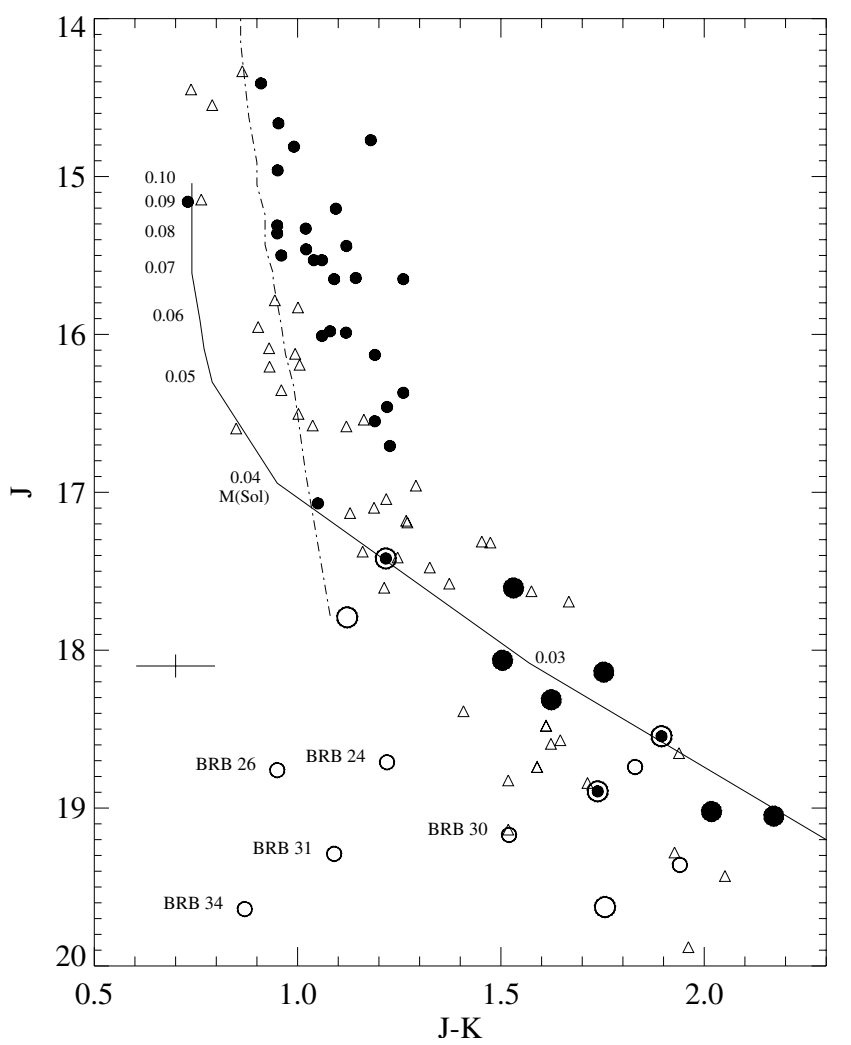

Fig. 5. $J$ versus $J-K$ colour-magnitude diagram, with the same definition of symbols and error bars as in Fig. 4. The small empty circles represent the $I J$ candidates without proper motion measurements.

NPL 40 (Festin 1998a,b) has a very blue $J-H$ colour, as noted by Pinfield et al. (2003). In Fig. 5, the brown dwarf CFHT-Pl-7, with its magnitude $J=15.16$ probably enhanced by a cosmic ray, is positioned at the left of the upper sequence, and the five very blue candidates at $J>18.5$ (empty circles) are probable contaminants.

In these near-IR colour-magnitude diagrams, where field dwarfs are also represented ( $\sim$ M3-L6), similar sequence overlaps appear at $J>17$ as in the $J$ versus $I-J$ diagram. Thus the sequence of the L brown dwarf candidates of the Pleiades is nearly indistinguishable from that of the field dwarfs shifted to the cluster distance. Note that the average errors in the distances of the sample field dwarfs are $\left\langle\sigma_{\mathrm{d}}\right\rangle=0.4 \mathrm{pc}$ (M5-M9) and $1.0 \mathrm{pc}$ (L0-L7), and the average errors in their translated $J$-band magnitudes, including the photometric errors and the distance error of $3 \mathrm{pc}$ for the Pleiades cluster, is of only 0.08 and $0.12 \mathrm{mag}$, respectively. The average error in their $I-J$ colours is $0.03 \mathrm{mag}$ (M5-M9) and $0.06 \mathrm{mag}$ (L0-L7). These magnitude and colour errors are small in comparison to the (field and cluster) trends observed.

From the $J$ versus $I-J, J-H$ and $J-K$ diagrams it appears that, opposite to the Pleiades late $\mathrm{M}$ dwarfs, many of the Pleiades L dwarf candidates have the same colours and absolute magnitudes as their field counterparts. This suggests that they may have the same spectral energy distributions and luminosities. If the effective temperatures estimated from the spectral energy distribution do not depend much on the gravity (as indicated by spectral synthesis), then, they may have also the same effective temperatures. These objects would therefore have the same radii. From Allen et al. (2005), the mean age of field L dwarfs is in the range 3-4 Gyr and the mean mass is in the range $0.06-0.07 M_{\odot}$, which according to the DUSTY models imply radii of $\sim 0.09 R_{\odot}$. 


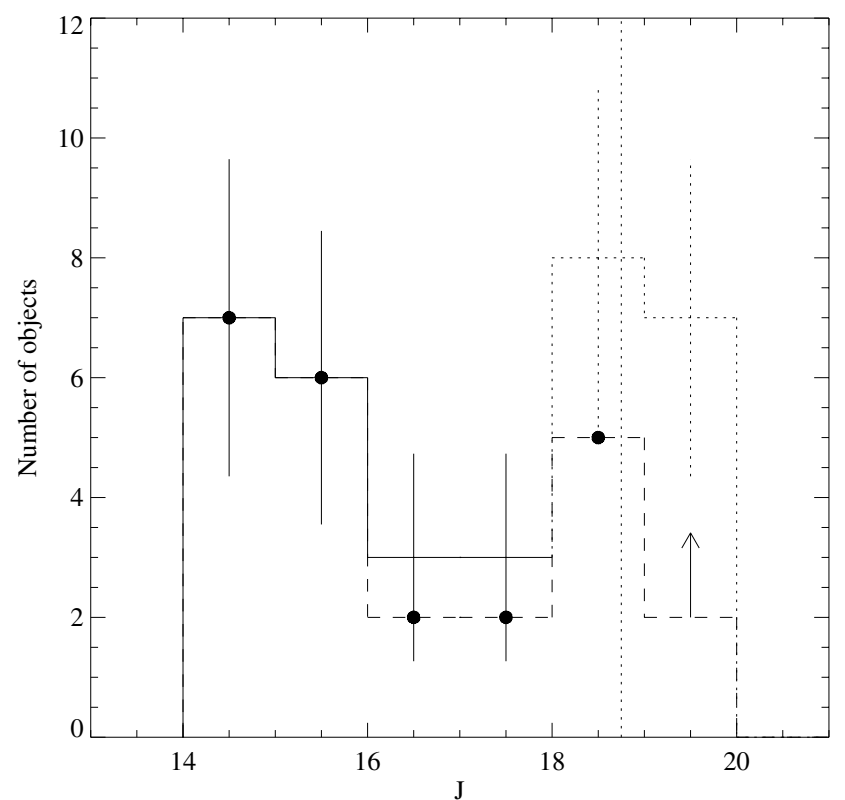

Fig. 6. Luminosity function for all the survey candidates (solid and dotted lines) and after contaminant correction (dashed line). The vertical dotted line represents the completeness limit of the survey.

These objects have almost reached the end of the contraction. We note however, that according to the same models (and using the luminosity estimates from Sect. 5), the radii of the Pleiades L brown dwarf candidates are of $\sim 0.125 R_{\odot}$, a value inconsistent with the previous one. Either the predicted evolution of radii with time may have to be revisited by the models, so the Pleiades $\mathrm{L}$ dwarfs reach radii of $0.09 R_{\odot}$ in about $120 \mathrm{Myr}$ or, the estimated masses and ages of the field $\mathrm{L}$ dwarfs may require a revision toward higher masses or smaller ages. A determination of the radii from the luminosities and the effective temperatures require precise spectroscopic observations which are beyond the scope of the present study.

\section{Substellar luminosity function}

The $J$-band luminosity function, the number of objects versus bin of $J$-band magnitude, is represented in Fig. 6 for BRB 1-34, our Pleiades very low-mass star and brown dwarf $I J$-band candidates $(J<18$ : solid line, $J>18$ : dotted line). The error bars are Poissonian errors, i.e. the square root of the number of candidates per bin. The vertical dotted line delimits the completeness of the survey, at $J \sim 18.75$. For this and fainter magnitudes, the present luminosity function is incomplete (dotted line).

\subsection{Contaminants}

The number of field $\mathrm{M}$ and $\mathrm{L}$ dwarfs that may contaminate our sample is obtained as follows. First we subdivide the region in the $J$ versus $I-J$ diagram occupied by the 34 objects of Table 1 in small rectangular sections. These sections have heights of half $J$-band magnitude and widths delimited by the bluer envelope (dashed line in Fig. 2) and the reddest object.

Then, we consider the typical $I-J$ colours of M- and L-type dwarfs as a function of the spectral type, based on the same sample of nearby cool dwarfs as mentioned in Sect. 3.2. Contaminants in our survey are essentially M5-L4 dwarfs. Using the relation from Dahn et al. (2002) between the spectral type of M7-L8 dwarfs and the maximum distance at which they can be observed for a given $J$-band magnitude (a relation that we assume also valid for M5 and M6 dwarfs), we compute for any spectral type contaminating a given rectangular section the pair of distances associated to the $J$-band magnitude boundaries of the section. Each pair of distances defines a volume towards the Pleiades, e.g. a truncated right pyramid with a square base, with top and base surfaces subtended by the survey angular area, $1.8 \mathrm{deg}^{2}$. This volume is located at the average of the pair of distances $(\langle d\rangle)$.

We use the densities of dwarfs in the solar neighbourhood provided by Kirkpatrick et al. (1994) for M5 and M6 spectral types, and by Cruz et al. (2003) for M7-L4 at $d<20$ pc. We take into account the completeness and sky coverage of these surveys. Chen et al. (2001) studied the star counts of the Sloan Digital Sky Survey for several hundred thousands stars at high latitudes north and south of the Galactic plane. They obtain an exponential scale height of the old thin disk of $330 \pm 3$ pc (late-type stars) and a Sun's distance from the plane of $27 \pm 4 \mathrm{pc}$. We assume that this scale height applies also for early L dwarfs. To compute the numerical density of a given spectral type dwarf at an average distance $\langle d\rangle$ from the Sun, we multiply the solar neighbourhood density by an exponential exp $-\left(\left|\langle d\rangle \sin \left(b_{\mathrm{Pl}}\right)-z_{\odot}\right| / z_{\mathrm{h}}\right)$, where $z_{\mathrm{h}}$ is the scale height, $z_{\odot}$ the Sun's distance to the Galactic plane, $b_{\mathrm{Pl}}=-23.52^{\circ}$ the Pleiades cluster's galactic latitude.

Finally we multiply the volumes by the corresponding densities and obtain the statistical numbers of contaminants. Each section in the $J$ versus $I-J$ diagram is thus contaminated by field dwarfs of different spectral types at different distances and with different densities. In Table 5, we summarize our estimate of contaminants. In the first column we indicate the $J$-mag range, in Col. " $N$ can", the number of $I J$ candidates (including CFHTPLIZ-25 and CFHT-PLIZ-25) and in Col. "Confirmed", the BRB numbers of the proper motion and lithium Pleiades members. In the next three columns, we give the estimated numbers of contaminants for the spectral type ranges M5-M7, M8-M9 and L0-L4. In Col. "total", the total number of contaminants is provided for the entire spectral type range M5-L4. Nine contaminants are already found (Col. "Contaminants found"): (i) CFHTPl-8 (M01), CFHT-PLIZ-25 (M03), BRB 19 and BRB 33; (ii) BRB 24, 26, 3031 and 34. The first set of objects is found by proper motion measurements and the second set by $J-K_{\mathrm{s}}$ photometry (Col. "Measurement"). The numbers of contaminants found in each spectral type range are indicated in parenthesis. Subtracting these from the total number of contaminants, we obtain the number of remaining contaminants that we may find among our survey candidates (Col. " $N$ r.c.").

After correcting the number of $I J$ candidates for contaminants in each $J$-mag range, we find an agreement with the number of confirmed Pleiades members, except for 15.0-16.0 and 19.0-20.0 mag. In these ranges one more contaminant than observed is predicted. For the latter range, this can be explained by its incompleteness. In both cases, we assume the observed number of confirmed members as the effective value when deriving the effective luminosity and mass functions, thus decreasing by one the value of $N_{\text {r.c. }}$. In Fig. 6 , the effective luminosity function is represented by the dashed histogram.

\section{Substellar mass function}

Finally, we present the implications of our results in the substellar mass function of the cluster surveyed area. The bolometric luminosity and effective temperature predictions of the DUSTY and NextGen models are mostly independent of the atmospheric properties (Chabrier et al. 2000), in contrast to the predicted 
Table 5. Contaminants among our low-mass star and substellar candidates.

\begin{tabular}{|c|c|c|c|c|c|c|c|c|c|}
\hline \multirow[t]{2}{*}{$J$ range } & \multirow[t]{2}{*}{$N_{\text {can }}{ }^{a}$} & \multirow{2}{*}{$\begin{array}{l}\text { Confirmed }{ }^{b} \\
\text { (Li or pm) }\end{array}$} & \multicolumn{4}{|c|}{ Estimated numbers of contaminants } & \multirow{2}{*}{$\begin{array}{l}\text { Contaminants } \\
\text { found }\end{array}$} & \multirow[t]{2}{*}{ Measurement } & \multirow[t]{2}{*}{$N_{\text {r.c. }}{ }^{c}$} \\
\hline & & & M5-M7 & M8-M9 & L0-L4 & total & & & \\
\hline $14.5-15.0$ & $7^{d}$ & $1,2,3,5,6,7$ & 0.3 & - & - & 0.3 & - & - & 0 \\
\hline $15.0-16.0$ & 7 & $8,9,10,11,12,13$ & $1.6(1)$ & - & - & 1.7 & CFHT-Pl-18 & prop. mot. & 1 \\
\hline $16.0-17.0$ & 3 & 14,15 & 0.8 & 0.3 & 0.1 & 1.2 & - & - & 1 \\
\hline $17.0-18.0$ & 4 & 17,18 & - & $1.3(2)$ & 0.3 & 1.6 & CFHT-PLIZ-25,BRB 19 & prop. mot. & 0 \\
\hline $18.0-19.0$ & 8 & $20,21,22,23,27$ & - & $1.7(2)$ & 1.3 & 3.0 & BRB 24, 26 & $J-K_{\mathrm{s}}$ & 1 \\
\hline$(19.0-20.0)$ & 7 & 28,29 & - & $2.0(2)$ & $3.8(2)$ & 5.9 & BRB 30, 31, 33, 34 & $J-K_{\mathrm{s}}$, prop. mot. & 2 \\
\hline
\end{tabular}

${ }^{a}$ Including CFHT-Pl-18 and CFHT-PLIZ-25.

${ }^{b}$ BRB number.

${ }^{c}$ We assume only 0 and 1 contaminants that remain to be found in the $J$ ranges $15.0-16.0$ and 19.0-20.0, respectively, when deriving the effective luminosity and mass functions (see text, Sect. 4.1).

${ }^{d}$ Including BRB 1 which has $J=14.41$.

Table 6. Mass bins for our mass function.

\begin{tabular}{ccccc}
\hline \hline$\Delta J$ & $\begin{array}{c}\Delta M \\
\left(M_{\odot}\right)\end{array}$ & $N_{\text {BRB }}$ & $N_{\text {cont }}$ & $N_{\text {eff }}$ \\
\hline $14.41-14.97$ & $0.147-0.104$ & 6 & 0 & 6 \\
$14.97-15.57$ & $0.104-0.075$ & 5 & 0 & 5 \\
$15.57-17.51$ & $0.075-0.039$ & 6 & 1 & 5 \\
$17.51-18.75$ & $0.039-0.026$ & 8 & 3 & 5 \\
$(18.75-19.65)$ & $0.026-0.018$ & 9 & 6 & 3 \\
\hline
\end{tabular}

magnitudes (e.g. for the M-L transition), which are not. We decided to infer a bolometric luminosity using a $J$-band bolometric correction and then to compare it with the model prediction for the distance and age of the Pleiades to obtain the mass.

Adopting the Pleiades cluster distance from Percival et al. (2005), we convert the $J$-band magnitudes to absolute magnitudes $M_{J}$, and add a bolometric correction $B C_{J}$ depending on $I-J$. This bolometric correction is obtained by Caballero (2006) from a fit to $\left(M_{\mathrm{bol}}-M_{J}, I-J\right)$ of the nearby cool dwarfs mentioned in Sect. 3.2. Binaries, peculiar dwarfs and dwarfs with line emission are not taken into account in the fit. For the nearby $M$ dwarfs, the bolometric magnitudes are from Golimowski et al. (2004), Leggett et al. (2000, 2002) and Dahn et al. (2002). For the nearby $\mathrm{L}$ and $\mathrm{T}$ dwarfs, these are mostly from Vrba et al. (2004), else from Leggett et al. (2002) and Geballe et al. (2001). The bolometric magnitudes $M_{\mathrm{bol}}=$ $M_{J}+B C_{J}$ that we obtained are then normalized to the solar value $M_{\text {bol, } \odot}=4.74$ (Livingston 2002) and converted to luminosities. Using these luminosities, we interpolate linearly the theoretical data points (DUSTY model for BRB 8-34 and NextGen model for BRB 1-7) and obtain the masses. These are grouped into five bins: two low-mass stellar and three substellar bins (see Table 6). The lowest mass bin is incomplete $(J \gtrsim 18.75)$. The differences between the masses derived from the bolometric luminosities of the NextGen- and DUSTY models for the fainter objects BRB 8-22, which extend into the lower luminosity range of the NextGen model, are smaller than $0.002 M_{\odot}$ and do not affect the distribution of objects in these bins. Each bin contains numbers of BRB candidates $\left(N_{\mathrm{BRB}}\right)$ and contaminants $\left(N_{\text {cont }}\right)$, identifiable from Tables 1 and 5. The width of the mass bins within the completeness of the survey is such that each bin contains approximately the same effective number of objects, $N_{\text {eff }}=N_{\text {BRB }}-N_{\text {cont }}$.

In Fig. 7, we show our results in the mass spectrum representation, $\log \mathrm{d} N / \mathrm{d} M, \log M$. Error bars refer to the Poissonian error. We show also the bins for the $\sim 50$ massive proper motion Pleiades objects from Deacon \& Hambly (2004) which

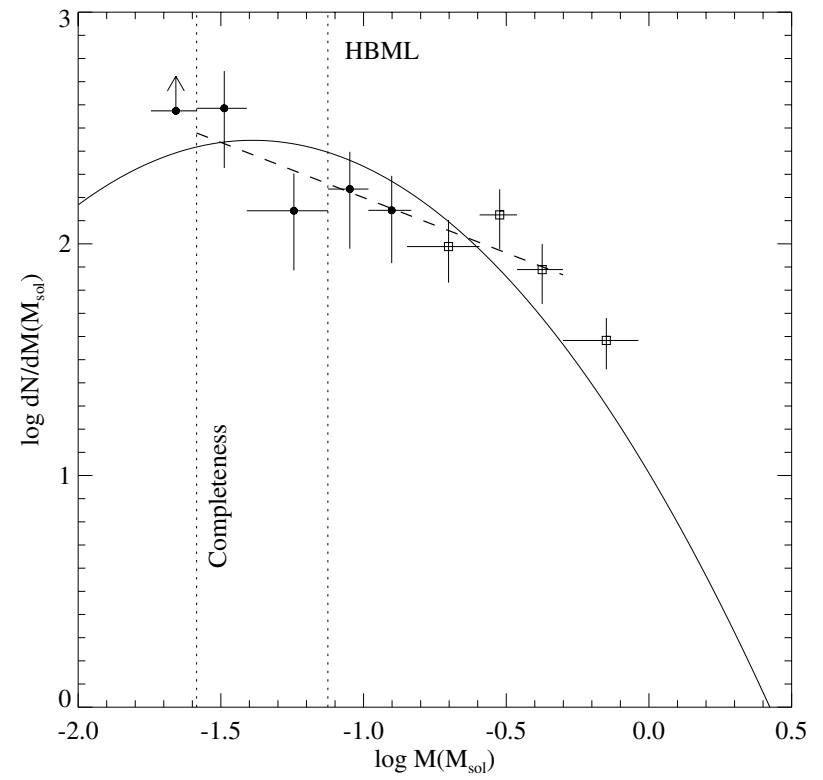

Fig. 7. The derived mass function for the Pleiades survey area. The filled circles are for the BRB objects, whereas the squares are for the objects from Deacon \& Hambly (2004). The right dotted line indicates the hydrogen-burning mass limit. The lower limit arrow for the lowest mass bin indicates that this bin is incomplete. The curve represents the log normal function obtained by Deacon \& Hambly (2004) scaled down to the total number of objects in the survey area and between $0.5 M_{\odot}$ and the completeness mass limit $0.026 M_{\odot}(56)$. The dashed line represents the power law fit to the data points between 0.5 and $0.026 M_{\odot}$, with $\alpha=0.5 \pm 0.2$.

are present in our fields ${ }^{3}$. For the $\sim 35$ fainter objects, corresponding essentially to $\mathrm{M}$ dwarfs ( $T_{\text {eff }} \lesssim 3600 \mathrm{~K}$ ), we obtain the masses with the method explained above, but using $I$ - and $J$-band magnitudes from Deacon \& Hambly (2004) and 2MASS, respectively, because these objects are saturated in our images. For the brighter ones we proceed similarly but, this time, using a synthetic $K$-band bolometric correction, function of the $(J-K)_{\text {CIT }}$ colour and determined by Houdashelt et al. (2000) for FGK stars with $4000 \mathrm{~K} \leq T_{\text {eff }} \leq 6500 \mathrm{~K}$. We assume solar metallicity and $\log g=4$. The CIT colour is transformed into the 2MASS equivalent (see the Explanatory Supplement to

3 The objects in common with our sample, DH 765 and DH 590, are taken into account only once and in our mass spectrum bins. 
the 2MASS All Sky Data Release ${ }^{4}$, Cutri et al. 2006) and the bolometric correction is applied on $2 \mathrm{MASS} J K_{\mathrm{s}}$-band photometry. For the objects in the K-M transition, the masses obtained by any of these two methods are within the highest mass bin ( $M>0.5 M_{\odot}$ ), thus fixing the number of objects in this bin. A linear fit to all the data points between 0.5 and $0.026 M_{\odot}$ provides an $\alpha=0.5 \pm 0.2$ for the power law $\mathrm{d} N / \mathrm{d} M \propto M^{-\alpha}$. It is compatible with the mass bin beyond the completeness limit of the survey (lower limit arrow at the left of the vertical dotted line, Fig. 7), defined by the three lowest mass possible or probable cluster members by proper motion, BRB 27, 28 and 29. Our estimate of $\alpha$ is in agreement with that from M03, $0.60 \pm 0.11$, and signifies that the number of objects per unit mass still increases at these low masses. In Fig. 7 the lognormal function obtained by Deacon \& Hambly (2004) is also represented, scaled down so that its integral over the mass range $0.5-0.026 M_{\odot}$ is equal to the number of objects in the survey area and in this mass range (56).

Our estimate of $\alpha$ is similar to those for younger clusters as $\alpha$ Per ( $~ 80 \mathrm{Myr})$, IC $4665(30-100 \mathrm{Myr})$ and $\lambda$ Orionis ( $\sim 5 \mathrm{Myr})$. Their values are: $0.56\left(0.2>M\left(M_{\odot}\right)>0.06\right.$; Barrado y Navascués et al. 2002), $0.6\left(1>M\left(M_{\odot}\right)>0.04\right.$; de Wit et al. 2006) and $0.60 \pm 0.06\left(0.86>M\left(M_{\odot}\right)>0.024\right.$; Barrado y Navascués et al. 2004), respectively. The presence of low-mass brown dwarfs after more than $100 \mathrm{Myr}$ in the Pleiades open cluster, in numbers relative to stars which are similar to those found in other younger clusters, implies that differential evaporation of low-mass members relative to more massive ones has not been very significant - in contrast to what may occur in the $\sim 600$ Myr old Praesepe open cluster (Chappelle et al. 2005). Comparison of observations to evolutionary models of the Pleiades cluster suggests indeed that only marginal differential evaporation of the massive brown dwarfs has occurred (Moraux et al. 2004; Moraux \& Clarke 2005). Therefore searches for the lowest mass brown dwarfs and even planetarymass objects should be conducted. A Pleiades giant planetarymass object at the deuterium-burning mass limit has a predicted effective temperature of $\sim 1300 \mathrm{~K}$ (DUSTY and COND models). This effective temperature corresponds to late $\mathrm{L}$ and early T field dwarfs (Golimowski et al. 2004) and magnitudes $J>20$ (Knapp et al. 2004) when scaled to the distance of the cluster. Such a free-floating object in the Pleiades open cluster may be detected in a photometric search with mid-size telescopes $(\sim 4 \mathrm{~m})$ but spectroscopic classification will require a very large telescope of 8-10 m class. The discovery of such low-mass Pleiades objects will permit to compare with free-floating low-gravity candidates in the solar neighbourhoud, as for example the T6 brown dwarf SDSS J111010.01+011613.1 (Knapp et al. 2004). Extrapolating the mass spectrum slope to lower masses, we find that our survey area may contain five brown dwarfs with masses $0.026-0.013 M_{\odot}$ and four planetary-mass objects with masses 0.013-0.05 $M_{\odot}$ (or two per square degree). In the case where the mass spectrum is log normal down to planetary masses, these values drop to three and one, respectively (or one planetary-mass object per square degree).

\section{Conclusions}

As a result of an $I J$-band survey in the Pleiades, we have found 18 brown dwarf candidates, most likely of L-type. They have $17.4<J<19.7, I-J>3.2$ and theoretical masses $\sim 0.040-0.020 M_{\odot}$. The near-IR follow-up for proper motion

4 http://www.ipac.caltech.edu/2mass/releases/allsky/ doc/explsup.html of 11 candidates permits us to confirm six as cluster members. Another three remain as possible members. Two are clear nonmembers. The near-IR photometric follow-up of the seven other candidates indicates that at least five are probable contaminants, because their $J-K_{\mathrm{s}}$ colours are bluer than those of the cluster proper motion members. These latter determine the L brown dwarf photometric sequence of the Pleiades. The sequence in the $J$ versus $I-J$ colour-magnitude diagram at $J>18$ is bluer than that predicted by the DUSTY atmospheres model, whereas it agrees with the predictions in the $J$ versus $J-H$ and $J-K$ diagrams. Moreover the sequence overlaps that of the field L dwarfs shifted to the cluster distance in the three colour-magnitude diagrams, suggesting that the Pleiades and field L dwarfs may have comparable spectral energy distributions and luminosities, and thus possibly similar radii.

We find evidence for an intrinsic velocity dispersion of Pleiades brown dwarfs at least four times that of Pleiades stars more massive than the Sun. The estimated value $>4$ mas $\mathrm{yr}^{-1}$ appears consistent with the kinematical expectations for brown dwarfs in a nearly relaxed cluster.

Correcting for contaminants and comparing with the proper motion objects from Deacon \& Hambly (2004) in the same fields, we obtain a substellar mass spectrum for the surveyed area of the cluster. For a power law $\mathrm{d} N / \mathrm{d} M \propto M^{-\alpha}$ fit in the mass range $0.5-0.026 M_{\odot}$, we find a spectral index $\alpha=0.5 \pm 0.2$ and in agreement with previous results. The slope is similar to that of much younger open clusters, suggesting the absence of significant differential evaporation of the low-mass brown dwarfs in the Pleiades. This supports deeper searches to detect lower mass free-floating objects in the cluster.

Acknowledgements. The $3.5 \mathrm{~m}$ Telescope is operated jointly by the Max-Planck Institut für Astronomie and the Instituto de Astrofísica de Andalucía (CSIC) at the Centro Astronómico Hispano Alemán (CAHA) at Calar Alto. The CanadaFrance-Hawaii Telescope (CFHT) is operated by the National Research Council of Canada, the Institut National des Sciences de l'Univers of the Centre National de la Recherche Scientifique of France, and the University of Hawaii. The William Hershel Telescope (WHT) is operated on the island of La Palma by the Isaac Newton Group in the Spanish Observatorio del Roque de los Muchachos of the Instituto de Astrofísica de Canarias. The Carlos Sánchez Telescope (TCS) is operated by the Instituto de Astrofísica de Canarias at the Teide Observatory, Tenerife. This publication makes use of data products from the Two Micron All Sky Survey, which is a joint project of the University of Massachusetts and the Infrared Processing and Analysis Center/California Institute of Technology, funded by the National Aeronautics and Space Administration and the National Science Foundation. We would like to thank J. Bouvier for allowing us to use the $R I$-band data and for refereeing this paper. We thank Terry Mahonay for the English corrections. The LIRIS observations were obtained with the assistance and help of the LIRIS commissioning team. We thank J. I. González Hernández and I. Villo for obtaining near-IR data for us, during LIRIS night 2005 January 24 and $\Omega 2000$ night 2005 February 1, respectively. We thank also N. Deacon, H. Bouy, E. Martín, M. R. Zapatero Osorio and E. Delgado-Donate for valuable discussions. J.A.P \& A.M. acknowledge the Plan Nacional de Astronomia y Astrofisica (AYA2004-03136), which supported part of this work.

\section{References}

Allen, P. R., Koerner, D. W., Reid, I. N., \& Trilling, D. E. 2005, ApJ, 625, 385 Baraffe, I., Chabrier, G., Allard, F., \& Hauschildt, P. H. 1998, A\&A, 337, 403 Barrado y Navascués, D., Bouvier, J., Stauffer, J. R., Lodieu, N., \& McCaughrean, M. J. 2002, A\&A, 395, 813

Barrado y Navascués, D., Stauffer, J. R., Bouvier, J., Jayawardhana, R., \& Cuillandre, J.-C. 2004, ApJ, 610, 1064

Basri, G. 2000, ARA\&A, 38, 485

Béjar, V. J. S. 2000, Ph.D. Thesis, Universidad de La Laguna

Béjar, V. J. S., Martín, E. L., Zapatero Osorio, M. R., et al. 2001, ApJ, 556, 830 Bihain, G., Rebolo, R., Béjar, V. J. S., et al. 2005, Astron. Nachr., 326, 1057 Bouvier, J., Stauffer, J. R., Martín, E. L., et al. 1998, A\&A, 336, 490 Bouy, H., Moraux, E., Bouvier, J., et al. 2006, ApJ, 637, 1056 Burgasser, A. J., Kirkpatrick, J. D., Brown, M. E., et al. 2002, ApJ, 564, 421 Caballero, J. A. 2006, Ph.D. Thesis, Universidad de La Laguna 
Chabrier, G., \& Baraffe, I. 2000, ARA\&A, 38, 337

Chabrier, G., Baraffe, I., Allard, F., \& Hauschildt, P. 2000, ApJ, 542, 464

Chappelle, R. J., Pinfield, D. J., Steele, I. A., Dobbie, P. D., \& Magazzù, A. 2005, MNRAS, 361, 1323

Chen, B., Stoughton, C., Smith, J. A., et al. 2001, ApJ, 553, 184

Cossburn, M. R., Hodgkin, S. T., Jameson, R. F., \& Pinfield, D. J. 1997, MNRAS, 288, L23

Cruz, K. L., Reid, I. N., Liebert, J., Kirkpatrick, J. D., \& Lowrance, P. J. 2003, AJ, 126, 2421

Cutri, R. M., Skrutskie, M. F., van Dyk, S., et al. 2003, VizieR Online Data Catalog, 2246, 0

Dahn, C. C., Guetter, H. H., Harris, H. C., et al. 2000, in ASP Conf. Ser., 212, From Giant Planets to Cool Stars, 74

Dahn, C. C., Harris, H. C., Vrba, F. J., et al. 2002, AJ, 124, 1170

de Wit, W. J., Bouvier, J., Palla, F., et al. 2006, A\&A, 448, 189

Deacon, N. R., \& Hambly, N. C. 2004, A\&A, 416, 125

Dobbie, P. D., Kenyon, F., Jameson, R. F., et al. 2002, MNRAS, 335, 687

Festin, L. 1998a, A\&A, 333, 497

Festin, L. 1998b, MNRAS, 298, L34

Geballe, T. R., Saumon, D., Leggett, S. K., et al. 2001, ApJ, 556, 373

Golimowski, D. A., Leggett, S. K., Marley, M. S., et al. 2004, AJ, 127, 3516

Hambly, N. C., Hawkins, M. R. S., \& Jameson, R. F. 1993, A\&AS, 100, 607

Hambly, N. C., Hodgkin, S. T., Cossburn, M. R., \& Jameson, R. F. 1999, MNRAS, 303, 835

Houdashelt, M. L., Bell, R. A., \& Sweigart, A. V. 2000, AJ, 119, 1448

Jameson, R. F., Dobbie, P. D., Hodgkin, S. T., \& Pinfield, D. J. 2002, MNRAS, 335,853

Jones, B. F. 1970, AJ, 75, 563

Kirkpatrick, J. D., McGraw, J. T., Hess, T. R., Liebert, J., \& McCarthy, D. W. 1994, ApJS, 94, 749

Kirkpatrick, J. D., Reid, I. N., Liebert, J., et al. 2000, AJ, 120, 447

Knapp, G. R., Leggett, S. K., Fan, X., et al. 2004, AJ, 127, 3553

Leggett, S. K., Allard, F., Dahn, C., et al. 2000, ApJ, 535, 965
Leggett, S. K., Golimowski, D. A., Fan, X., et al. 2002, ApJ, 564, 452 Livingston, W. C. 2002, in Allen's Astrophysical Quantities, ed. A. N. Cox (4th ed.; New York: Springer), 151

Martin, E. L., Rebolo, R., \& Zapatero-Osorio, M. R. 1996, ApJ, 469, 706 Martín, E. L., Basri, G., Gallegos, J. E., et al. 1998a, ApJ, 499, L61

Martín, E. L., Basri, G., Zapatero Osorio, M. R., Rebolo, R., \& López, R. J. G. 1998b, ApJ, 507, L41

Martín, E. L., Delfosse, X., Basri, G., et al. 1999, AJ, 118, 2466

Martín, E. L., Brandner, W., Bouvier, J., et al. 2000, ApJ, 543, 299

Moraux, E., \& Clarke, C. 2005, A\&A, 429, 895

Moraux, E., Bouvier, J., \& Stauffer, J. R. 2001, A\&A, 367, 211

Moraux, E., Bouvier, J., Stauffer, J. R., \& Cuillandre, J.-C. 2003, A\&A, 400, 891

Moraux, E., Kroupa, P., \& Bouvier, J. 2004, A\&A, 426, 75

Nagashima, C., Dobbie, P. D., Nagayama, T., et al. 2003, MNRAS, 343, 1263

Padoan, P., \& Nordlund, A.. 2004, ApJ, 617, 559

Percival, S. M., Salaris, M., \& Groenewegen, M. A. T. 2005, A\&A, 429, 887

Perryman, M. A. C., Lindegren, L., Kovalevsky, J., et al. 1997, A\&A, 323, L49

Pinfield, D. J., Jameson, R. F., \& Hodgkin, S. T. 1998, MNRAS, 299, 955

Pinfield, D. J., Hodgkin, S. T., Jameson, R. F., et al. 2000, MNRAS, 313, 347

Pinfield, D. J., Dobbie, P. D., Jameson, R. F., et al. 2003, MNRAS, 342, 1241

Rebolo, R., Zapatero Osorio, M. R., \& Martín, E. L. 1995, Nature, 377, 129

Rebolo, R., Martín, E. L., Basri, G., Marcy, G. W., \& Zapatero Osorio, M. R. 1996, ApJ, 469, L53

Robichon, N., Arenou, F., Mermilliod, J.-C., \& Turon, C. 1999, A\&A, 345, 471 Schwartz, M. J., \& Becklin, E. E. 2005, AJ, 130, 2352

Stauffer, J. R., Schild, R., Barrado y Navascues, D., et al. 1998a, ApJ, 504, 805 Stauffer, J. R., Schultz, G., \& Kirkpatrick, J. D. 1998b, ApJ, 499, L199

Tej, A., Sahu, K. C., Chandrasekhar, T., \& Ashok, N. M. 2002, ApJ, 578, 523

Vrba, F. J., Henden, A. A., Luginbuhl, C. B., et al. 2004, AJ, 127, 2948

Zapatero Osorio, M. R., Rebolo, R., \& Martín, E. L. 1997a, A\&A, 317, 164

Zapatero Osorio, M. R., Rebolo, R., Martín, E. L., et al. 1997b, ApJ, 491, L81

Zapatero Osorio, M. R., Rebolo, R., Martín, E. L., et al. 1999, A\&AS, 134, 537 